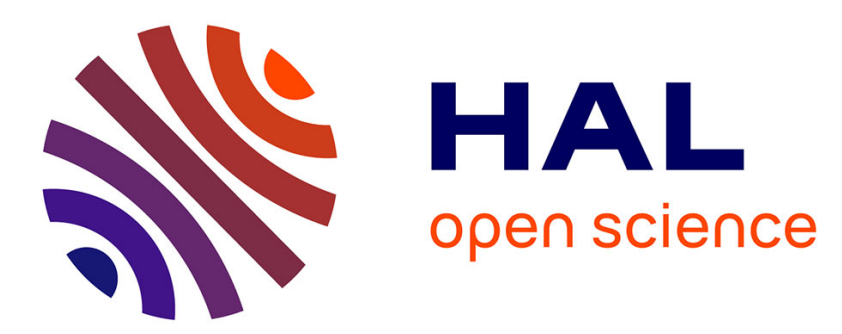

\title{
Empirical Mode Decomposition: Applications on signal and image processing
}

Jean-Claude J. C. Nunes, Eric Deléchelle

\section{To cite this version:}

Jean-Claude J. C. Nunes, Eric Deléchelle. Empirical Mode Decomposition: Applications on signal and image processing. Advances in Adaptive Data Analysis, 2009, 1 (1), pp.125-175. 10.1142/S1793536909000059 . hal-00665784

\section{HAL Id: hal-00665784 \\ https://hal.science/hal-00665784}

Submitted on 3 Feb 2012

HAL is a multi-disciplinary open access archive for the deposit and dissemination of scientific research documents, whether they are published or not. The documents may come from teaching and research institutions in France or abroad, or from public or private research centers.
L'archive ouverte pluridisciplinaire HAL, est destinée au dépôt et à la diffusion de documents scientifiques de niveau recherche, publiés ou non, émanant des établissements d'enseignement et de recherche français ou étrangers, des laboratoires publics ou privés. 


\title{
EMPIRICAL MODE DECOMPOSITION: APPLICATIONS ON SIGNAL AND IMAGE PROCESSING
}

\author{
JEAN-CLAUDE NUNES* ${ }^{*}$ and ÉRIC DELÉCHELLE ${ }^{\dagger}$ \\ *Laboratoire Traitement du Signal et de l'Image (LTSI, UMR INSERM 642) \\ Campus de Beaulieu, Btiment 22, Université de Rennes 1 \\ 35042 Rennes Cedex - France \\ ${ }^{\dagger}$ Laboratoire Images, Signaux et Systèmes Intelligents (LISSI, EA 3956) \\ Université Paris XII - Val de Marne \\ 61, avenue du Général de Gaulle \\ 94010 Créteil cedex, France \\ *jean-claude.nunes@univ-rennes1.fr
}

In this paper, we propose some recent works on data analysis and synthesis based on Empirical Mode Decomposition (EMD). Firstly, a direct 2D extension of original Huang EMD algorithm with application to texture analysis, and fractional Brownian motion synthesis. Secondly, an analytical version of EMD based on PDE in 1D-space is presented.

We proposed an extension in 2D-case of the so-called "sifting process" used in the original Huang's EMD. The 2D-sifting process is performed in two steps: extrema detection (by neighboring window or morphological operators) and surface interpolation by splines (thin plate splines or multigrid B-splines). We propose a multiscale segmentation approach by using the zero-crossings from each 2D-intrinsic mode function (IMF) obtained by 2D-EMD.

We apply the Hilbert-Huang transform (which consists of two parts: (a) Empirical mode decomposition, and (b) the Hilbert spectral analysis) to texture analysis. We analyze each 2D-IMF obtained by 2D-EMD by studying local properties (amplitude, phase, isotropy, and orientation) extracted from the monogenic signal of each one of them. The monogenic signal proposed by Felsberg et al. is a 2D-generalization of the analytic signal, where the Riesz transform replaces the Hilbert transform. These local properties are obtained by the structure multivector such as proposed by Felsberg and Sommer.

We present numerical simulations of fractional Brownian textures. Recent works published by Flandrin et al. relate that, in the case of fractional Gaussian noise (fGn), EMD acts essentially as a dyadic filter bank that can be compared to wavelet decompositions. Moreover, in the context of fGn identification, Flandrin et al. show that variance progression across IMFs is related to Hurst exponent $H$ through a scaling law. Starting with these results, we proposed an algorithm to generate fGn, and fractional Brownian motion (fBm) of Hurst exponent $H$ from IMFs obtained from EMD of a White noise, i.e., ordinary Gaussian noise (fGn with $H=1 / 2$ ). 
Deléchelle et al. proposed an analytical approach (formulated as a partial differential equation (PDE)) for sifting process. This PDE-based approach is applied on signals. The analytical approach has a behavior similar to that of the EMD proposed by Huang.

Keywords: Empirical mode decomposition; Hilbert-Huang transform; Riesz transform; monogenic signal; texture analysis; nonlinear partial differential equation.

\section{Introduction}

This paper describes the formulation and application of the empirical mode decomposition (EMD) in data analysis or synthesis. We present our work which have been published in Refs. 22, 23 and 74-76.

Firstly, we extend the EMD to 2D-data (i.e., images), the so-called bidimensional EMD (BEMD), the process being called 2D-sifting process (published in Refs. 74 and 75 ). The 2D-sifting process is performed in two steps: extrema detection by neighboring window or morphological operators (morphological reconstruction or watershed) and surface interpolation by splines (thin plate splines or multigrid B-splines). Moreover, we detect the zero crossings of each 2D-intrinsic mode function (IMF) and suggest a multiscale segmentation from these zero crossings (such as proposed in Ref. 74).

Secondly, we present numerical simulations of fractional Brownian motion (1D and 2D) from EMD of ordinary Gaussian noise (fGn with $H=1 / 2$ ) (published in Ref. 23).

Thirdly, we analyze each 2D-IMF obtained by 2D-EMD by studying local properties (amplitude, phase, isotropy, and orientation) extracted from the monogenic signal of each one of them (published in Ref. 76). The monogenic signal is a 2D-generalization of the analytic signal, where the Riesz transform replaces the Hilbert transform.

Fourthly, we propose an analytical approach of the EMD. Indeed, we replace the sifting process by nonlinear partial differential equation (PDE) (published in Ref. 22). The analytical approach has a behavior similar to that of the EMD proposed by Huang.

\section{Bidimensional Empirical Mode Decomposition (BEMD)}

The Hilbert-Huang transform, first introduced by Huang et al. ${ }^{51}$ consists of two parts: (1) empirical mode decomposition (EMD), and (2) Hilbert spectral analysis. As different applications as medical and seismic signals analyses have shown the effectiveness of this time-frequency analysis method. This method permits analyzing 1D-nonlinear and nonstationary data. Comparisons with wavelet and Fourier analyses show that the Hilbert-Huang transform offers much better temporal and frequency resolutions. ${ }^{52}$ We propose here an extension of the EMD to 2D-data. 


\subsection{Empirical Mode Decomposition (EMD)}

The EMD is locally adaptive and suitable for analysis of nonlinear or nonstationary processes. The starting point of EMD is to consider oscillatory signals at the level of their local oscillations and to formalize the idea that:

$$
\text { "signal = fast oscillations surimposed to slow oscillations" }
$$

and to iterate on the slow oscillation components considered as a new signal. This one-dimensional decomposition technique extracts a finite number of oscillatory components or "well-behaved" AM-FM functions, called intrinsic mode function (IMF), directly from the data.

The IMFs are obtained from the signal by means of an algorithm called the sifting process. The sifting procedure is based on two constraints: each IMF has the same number of zero-crossings and extrema, and also has symmetric envelopes defined by the local maxima, and minima, respectively. Furthermore, it assumes that the signal has at least two extrema. The sifting algorithm is represented in Fig. 1.

So, for any one-dimensional discrete signal $I_{\text {ori }}$, EMD can finally be presented with the following representation:

$$
I_{\text {ori }}=\sum_{j=0}^{J} I_{\text {mode }}(j)+I_{\text {res }}
$$

where $I_{\text {mode }}(j)$ is the $j$ th mode (or IMF) of the signal, and $I_{\text {res }}$ is the residual trend (a low-order polynomial component). The sifting procedure generates a finite (and limited) number of IMFs that are nearly orthogonal to each other. ${ }^{51}$

\subsection{State of the art of the BEMD}

We were one of very first to suggest an implementation of 2 D-EMD. ${ }^{74,75}$ Starting from this 2D-EMD, we proposed various methods of analysis and synthesis of images. ${ }^{23,76}$ Works also relating to this field were proposed by other research teams in image processing:

- texture analysis, ${ }^{97}$

- fractal analysis, ${ }^{64,65}$

- image compression. ${ }^{61-63}$

Linderhed ${ }^{61-63}$ proposes decompositions based on two surface interpolations: bi-cubic splines or thin plate splines (TPS). Nunes et al. ${ }^{23,74-76}$ propose decompositions based on two surface interpolations by splines: TPS or multilevel B-splines. Damerval et al. ${ }^{21}$ propose a decomposition based on Delaunay triangulation and on piecewise cubic polynomial interpolation. The method proposed by Liu et al. ${ }^{64}$ carry out an EMD according to only one direction and not a true 2D-EMD. Indeed, this method divides a two-dimensional data (or image) into one-dimensional data. The 1D-EMD is applied on a limited number of directions: two (horizontal and vertical) or more. Such approach shows quickly its limits in the case of local image analysis. 


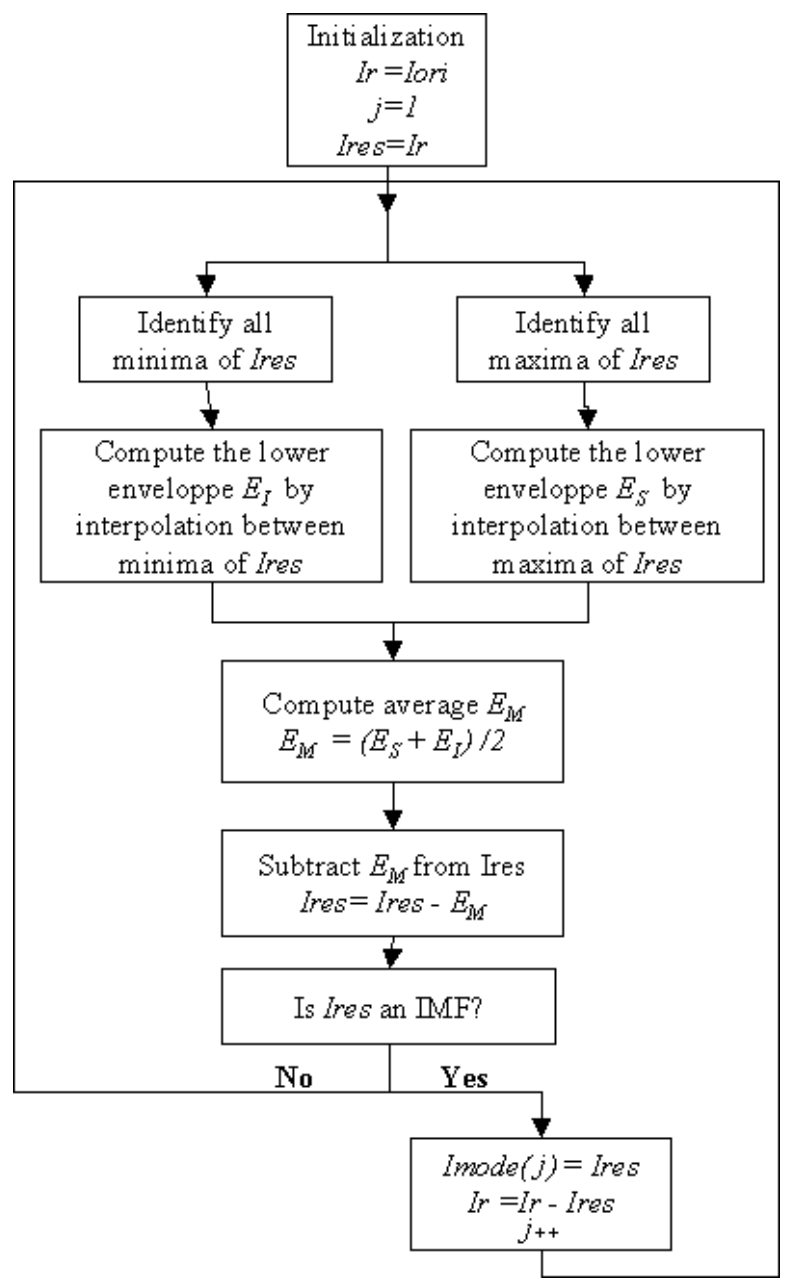

Fig. 1. The sifting process.

Except in Ref. 74 few results were published concerning multiscale dyadic representations obtained by BEMD of real, textured or medical images. Moreover, no comparison between the various methods was carried out. In Ref. 74, the behavior of the BEMD was described: almost dyadic behavior by the layout of the impulse response and the layout of the decrease of the extrema number.

\subsection{Our BEMD approach}

We present here our work which was published in Refs. 74- 76. A 2D-IMF obtained by $2 \mathrm{D}$-sifting process is 2D- "well-behaved" AM-FM function. To extract the 2DIMF during the $2 \mathrm{D}$-sifting process, ${ }^{74}$ we have used neighboring window or morphological operators to detect the image extrema and radial basis functions or multilevel B-splines to compute the surface interpolation. 


\subsubsection{Extremal point detection}

To detect the image extrema, we used neighboring window or morphological operators (morphological reconstruction and watershed).

Neighboring window: We locate extrema points (maxima and minima) of the image intensity function within a neighboring window. Here, the extrema have values strictly higher or lower than all their closer neighbors (4, 6 or 8 connexity). The extrema are found only when they have a size of one pixel. We choosed eight connexity.

Morphological reconstruction: Moreover, we used morphological reconstruction to find extremal plateaus. Morphological reconstruction ${ }^{13}$ finds regions of uniform pixel value whose neighboring regions all have smaller or larger values (maximal or minimal plateaus). Since, the extrema definition by mathematical morphology is more precise, the detection of the extrema is carried out by morphological reconstruction based on geodesic operators. We define the geodesic reconstruction as follows.

The grayscale reconstruction $\operatorname{Irec}_{I}(M)$ of $I$ from $M$ is obtained by iterating grayscale geodesic dilations of $M$ under $I$ until a stability is reached; that is,

$$
\operatorname{Irec}_{I}=\underset{n \geq 1}{\vee} \partial_{1}^{n}(M) .
$$

If we take $M=I-1$ (subtract one gray level from every pixel of original image) and if we perform the reconstruction Irec (by geodesic dilation) of $M$ by $I$, the difference $I-$ Irec corresponds to the indicator function of the maxima of $I$.

Conversely, the difference between Irec ${ }^{*}$ (reconstruction by geodesic erosion) and $I$ (original image) produces the indicator function of the minima of $I$. We used this extrema detection method which is described in Ref. 94.

Crest or valleys line detection by morphological operator (i.e., watershed): In Ref. 74, the author proposes to interpolate not by means of the extrema but by means of the peak lines or the valley lines by using the watershed (morphological operator).

Watershed segmentation draws its origins from mathematical morphology, and is in fact a region growing algorithm that treats the input image as a topographic surface, and through the intuitive process of water-filling, creates a partition of the image. For the implementation of the watershed algorithm, we used a fast method suggested in Ref. 94.

By flooding the topographic surface of an image from its regional minimum and preventing the merging of water coming from different sources, we partition the image into two different sets; the catchment basins and the watershed lines, where each catchment basin contains one and only one regional minimum. ${ }^{12}$

At the contact lines the "watershed lines" are created and constitute the limits between the image regions. ${ }^{12}$ 
The watershed of a surface has some very useful properties:

- the watershed lines form closed simply connected regions;

- all points belong to a region or fall on a watershed;

- each regional minimum possesses a single catchment basin (region) associated with it.

These watershed lines mark the crest lines of the original image. To obtain the lines of valleys, we reverse the levels of gray of the image.

Results of extrema detection: Figure 2 shows the maxima detection from three methods (neighboring window, morphological reconstruction and watershed). The BEMD obtained from these three types of extrema by neighboring window,

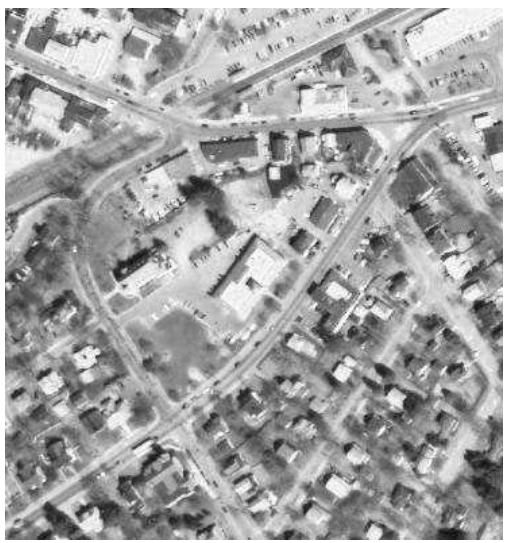

(a)

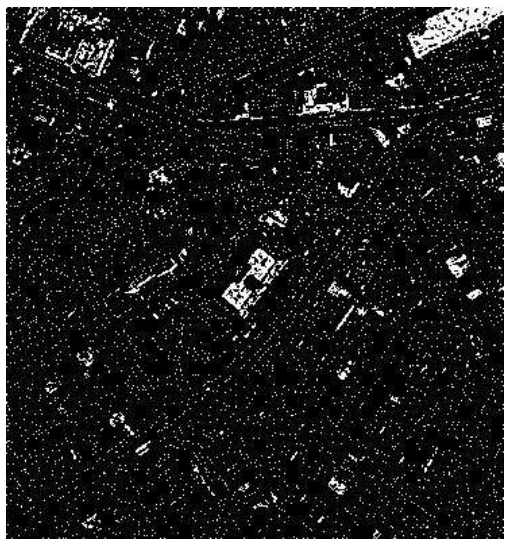

(c)

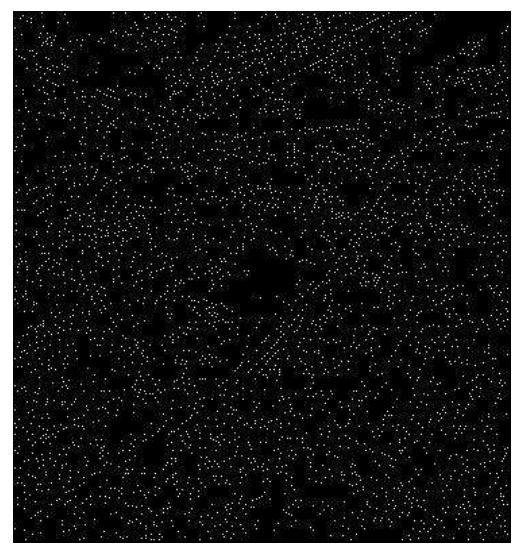

(b)

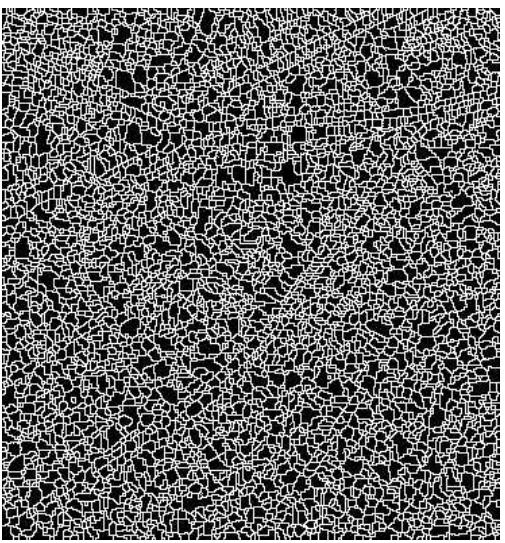

(d)

Fig. 2. Maxima detection by three methods (neighboring window, morphological reconstruction, and watershed) of aerial image. (a) Original image, (b) Maxima obtained by neighboring window, (c) Maxima obtained by morphological reconstruction and (d) Maxima obtained by watershed. 
morphological reconstruction, and watershed are all different. Indeed, overdecomposition in the case of BEMD from crest and valley lines is noted. ${ }^{74}$

\subsubsection{Scattered data interpolation}

There exist many solutions to scattered data interpolation which include Shepard's methods, ${ }^{85}$ radial basis functions, ${ }^{48}$ and finite element methods. There is a vast amount of literature devoted to scattered data interpolation. Readers are referred to Refs. 2, 6, 42, 44, 50, 66, 73 for excellent surveys. In the case of BEMD, we compute surface interpolation by radial basis functions (TPS) or by multilevel B-splines from extrema points.

Surface interpolation by radial basis functions: According to Franke, ${ }^{42}$ radial basis functions $(\mathrm{RBF})$ are known to be one of the sharpest and stablest methods to solve scattered data approximation and interpolation problems. ${ }^{14}$ The initial ideas, dating from the late 1960s, are due to Hardy. ${ }^{48}$ RBF methods are some of the most elegant schemes from a mathematical point of view. ${ }^{42}$

An $\mathrm{RBF}$ is a function of the form:

$$
s(x)=p_{m}(x)+\sum_{i=1}^{N} \lambda_{i} \Phi\left(\left\|x-x_{i}\right\|\right),
$$

with $x \in \Re^{d}, \lambda_{i} \in \Re$, where

- $s$ is the RBF,

- $p_{m}$ is a low-degree polynomial, typically linear or quadratic, a member of the $m$ th degree polynomials in $d$ variables,

- $\|\cdot\|$ denotes the Euclidean norm,

- the $\lambda_{i}$ 's are the RBF coefficients,

- $\Phi$ is a real-valued function called the basis function, and

- the $x_{i}$ 's are the RBF centers.

Classical examples for these functions are given by Duchon's thin plate splines $^{26}$ and Hardy's multiquadratics, ${ }^{48}$ where developments during this decade have provided compactly supported RBF (CSRBF) and RBFs with multizone decomposition.

A variety of different RBFs have been proposed. These are, for example, linear (L), cubic (C), thin-plate splines (TPS), multiquadrics (M), inverse multiquadrics (IM), and the Gaussian (G):

$$
\begin{gathered}
\Phi_{\mathrm{L}}(r)=r, \\
\Phi_{\mathrm{C}}(r)=r^{3}, \\
\Phi_{\mathrm{TPS}}(r)=r^{2} \log r, \\
\Phi_{\mathrm{M}}(r)=\sqrt{1+(\epsilon r)^{2}},
\end{gathered}
$$




$$
\begin{gathered}
\Phi_{\mathrm{IM}}(r)=\frac{1}{\sqrt{1+(\epsilon r)^{2}}}, \\
\Phi_{\mathrm{G}}(r)=e^{-(\epsilon r)^{2}} .
\end{gathered}
$$

Drawbacks of this method are that:

- the far field expansion has to be done separately for every basis function, and is very complex to implement;

- the required computational work is proportional to the number of grid nodes and the number of scattered data points. Special methods for reducing the processing time were developed for TPS, and were discussed in Refs. 8, 17, 60. In spite of significant progress in the field of implementing RBFs and CSRBFs ${ }^{56,71}$ for reconstruction purposes, it is still an open question whether it is possible to handle realistic amounts of data in real time.

We computed surface interpolation by TPS through a set of irregularly spaced points (extrema) during the 2D-sifting process. ${ }^{23,74-76}$

Surface interpolation with multilevel B-splines: Splines are extensively used in image processing, primarily in interpolation tasks due to their approximation properties. From among spline properties beneficial to image processing, we should mention: compact support, existence of fast algorithms to compute coefficients from function values and vice versa, and the facility to compute derivatives as spline functions of lower degree. B-splines are very easy to manipulate and have been used widely and successfully used in Computer-Aided Geometric Design, computer graphics and visualization. ${ }^{7}$ The mathematical description of the B-spline interpolation problem can be found in many textbooks, tutorials and papers, such as Refs. 91-93, or others. The standard approach is to solve a sparse linear equations system. There are several types of splines that can be used. The simplest approach is to consider tensor product splines ${ }^{41}$ and their generalizations to non-uniform rational B-splines (NURBS) surfaces, ${ }^{81}$ which have important applications, e.g., in modeling and designing surfaces. These spaces are essentially restricted to rectangular domains.

Several multilevel methods for surface construction have been studied and presented over the past years, although relatively few deal with approximation of scattered data.

Multilevel B-splines, proposed in Ref. 59, are introduced to compute a $C 2$ continuous surface through a set of irregularly spaced points using a coarse-to-fine hierarchy of control lattices. The method is local in the sense that on the finer tensor product grids each B-spline coefficient is computed from nearby points only. Consequently, the method is also fast.

Other spline methods are based on box splines, ${ }^{20,49}$ simplex splines, ${ }^{100}$ splines of finite-element type, or approaches due to multiresolution analysis and wavelets. ${ }^{35}$ 
Many authors have investigated multiresolution analysis for manipulating spline surfaces and polygonal meshes. ${ }^{10,11,18}$

We used multilevel B-splines (described in Ref. 59) for scattered data interpolation during the 2D-sifting process. ${ }^{74}$ Multilevel B-spline interpolation, presented in Ref. 59, is a hierarchical method that first tries to fit a set of globally smooth B-splines through the sampled data each defined on a distinct $4 * 4$ grid of control points with large coverage. In each successive step the number of control point in the grid of each B-spline is doubled in each direction and a new set of B-splines is created on the four smaller $4 * 4$ grids of control points. These new B-splines on the smaller grids are fitted through the difference of the sum of the already computed B-splines.

According to Bertram et al. ${ }^{10}$ a drawback of this method is that the global refinement of lattices is inefficient when accurately reproducing small local features.

\subsubsection{Stopping criteria}

We have to determine a criterion for the 2D-sifting process to stop. This can be accomplished by limiting the size of the standard deviation (SD), computed from the two consecutive sifting results $\operatorname{Ires}_{j(i-1)}$ and $\operatorname{Ires}_{j i}$ for the $j$ th mode as:

$$
\mathrm{SD}=\sum_{k=0}^{K} \sum_{l=0}^{L}\left[\frac{\left|\left(\operatorname{Ires}_{j(i-1)}(k, l)-\operatorname{Ires}_{j i}(k, l)\right)\right|^{2}}{\operatorname{Ires}_{j(i-1)}^{2}(k, l)}\right] .
$$

We added two conditions with this stopping criteria:

- the iteration count to obtain an IMF is lower or equal to a constant. At the end of 12 iterations, we admit that the residual image Ires $_{j i}$ is an IMF,

- we also obtain an IMF when the difference between two consecutive $\mathrm{SD}\left(\mathrm{SD}_{i}\right.$ and $\mathrm{SD}_{i}$ ) is lower than a threshold (precision $* \mathrm{SD}_{i}$ ). We calculate it this way. If $\left(\mathrm{SD}_{i}-\mathrm{SD}_{i-1}\right)<\left(\right.$ precision $\left.* \mathrm{SD}_{i}\right)$, the residual image Ires $_{j i}$ is an IMF.

\subsection{BEMD results and discussion}

Firstly, we describe the 2D-sifting process. Secondly, we show nonlinear multiscale representation of images obtained by BEMD.

\subsubsection{Multiscale representation and BEMD}

The 2D-decomposition by sifting process of an image provides a representation that is easy to interpret. Examples using images $(512 \times 512$ in grayscale) (Figs. 3-8, 9 and 10) from the Brodatz texture album or photographies are shown in Figs. 11-13, 16-22, 54 and 55. To stop the sifting process, we have used the standard deviation (SD). We have used SD smaller than 1.

We proposed in Figs. 17-19 BEMD of landshaft image (Fig. 7) by TPS from three extrema detection method. 


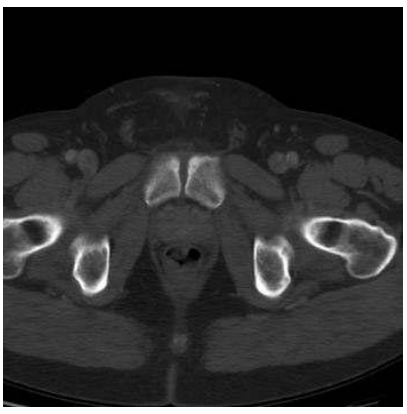

Fig. 3. Bassin image.

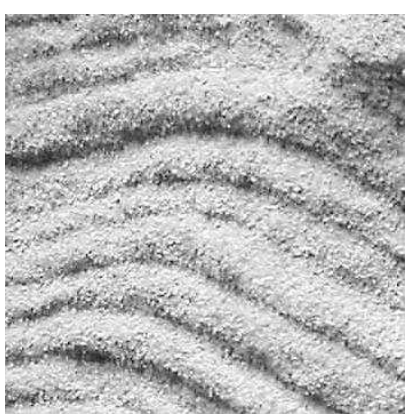

Fig. 4. Abbey texture.

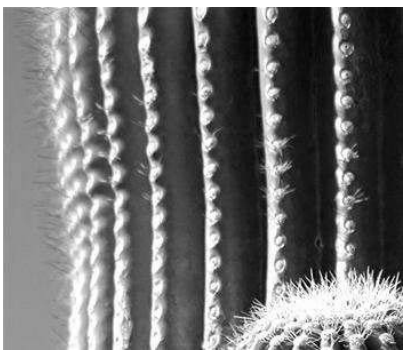

Fig. 5. Cactus.

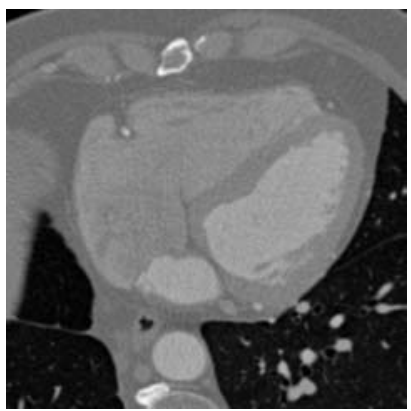

Fig. 6. Cardiac image.

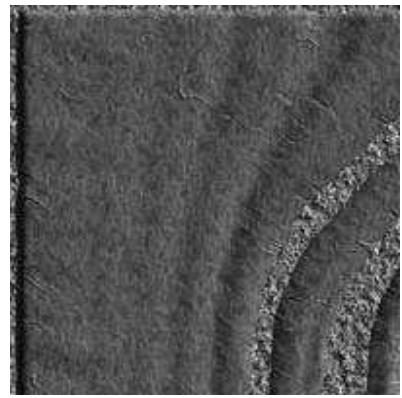

Fig. 7. Landshaft image.

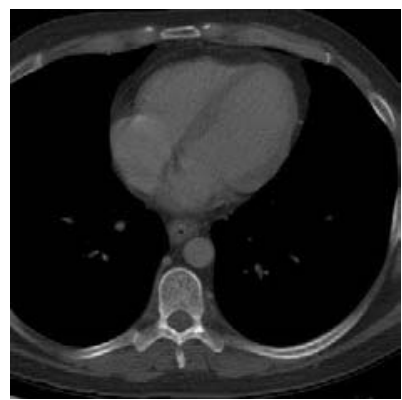

Fig. 8. Heart image.

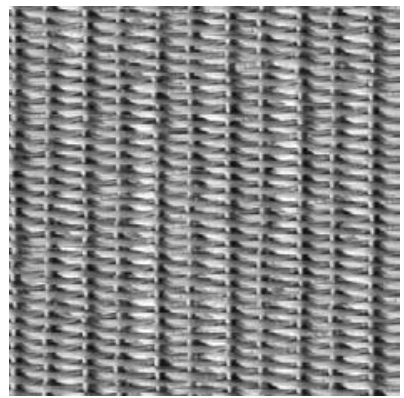

Fig. 9. Fabric image.

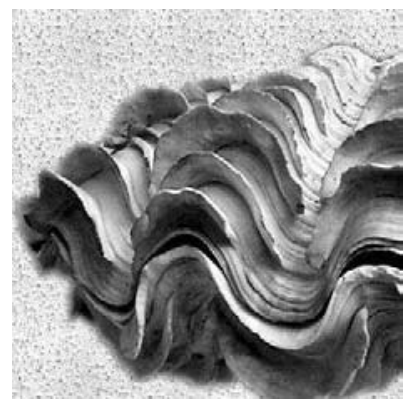

Fig. 10. Wavy image. 

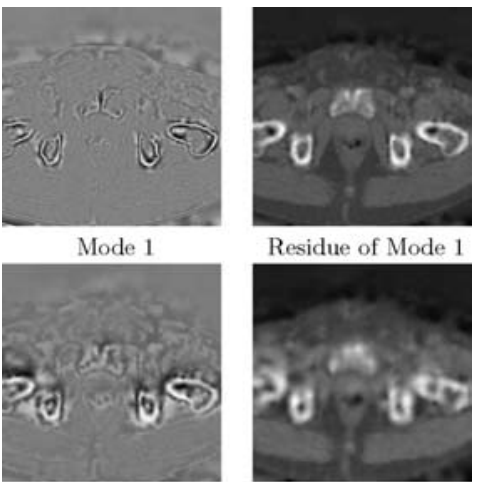

Mode 2

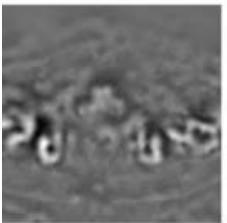

Mode 3

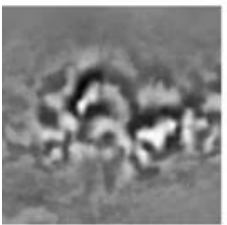

Mode 4

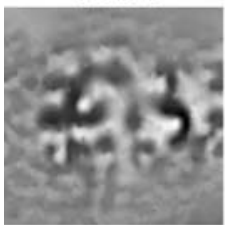

Mode 5

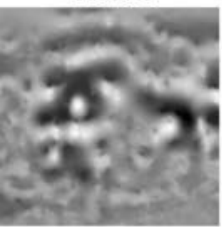

Mode 6

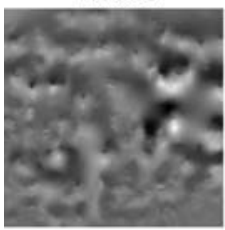

Mode 7

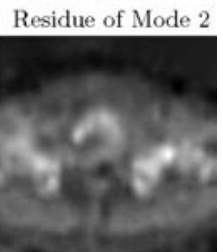

Residue of Mode 3

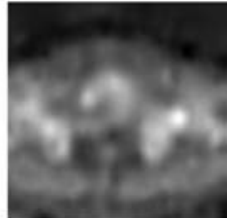

Residue of Mode 4

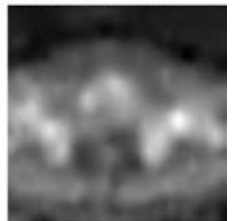

Residue of Mode 5

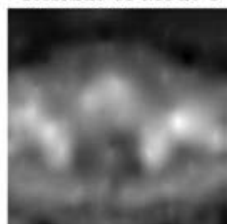

Residue of Mode 6

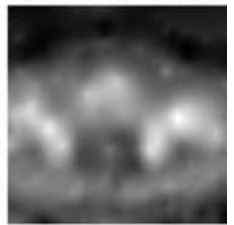

Residue of Mode 7

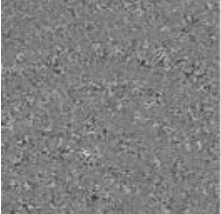

Mode 1

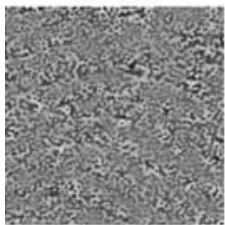

Mode 2

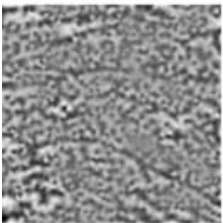

Mode 3

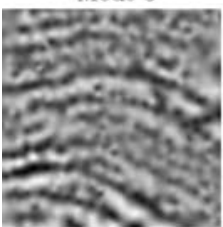

Mode 4

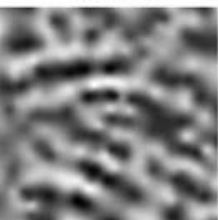

Mode 5

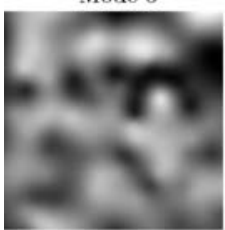

Mode 6

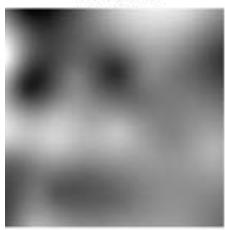

Mode 7

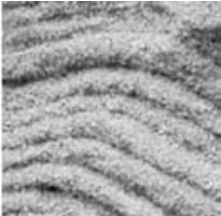

Residue of Mode 1

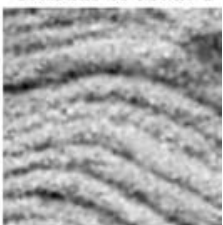

Residue of Mode 2

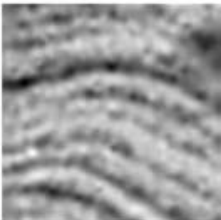

Residue of Mode 3

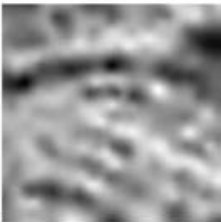

Residue of Mode 4

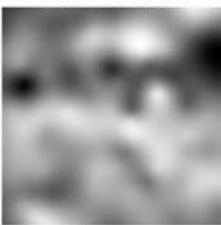

Residue of Mode 5

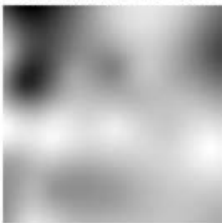

Residue of Mode 6

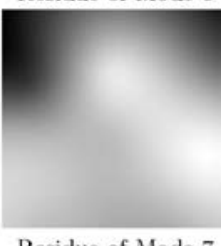

Residue of Mode 7
Fig. 11. BEMD of Bassin image (Fig. 3) by TPS from extrema obtained by morphological reconstruction.
Fig. 12. BEMD of abbey texture (Fig. 4) by TPS from extrema obtained by morphological reconstruction. 


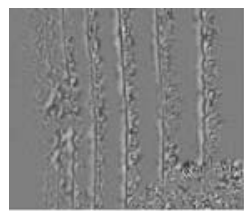

Mode 1

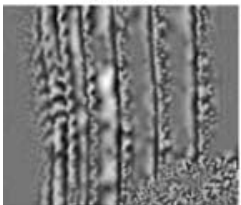

Mode 2

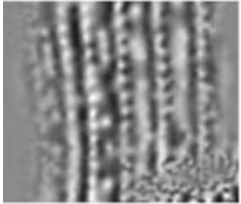

Mode 3

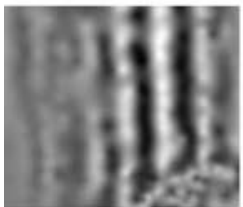

Mode 4

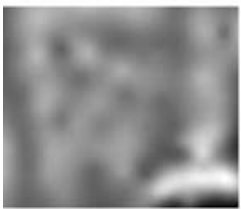

Mode 5

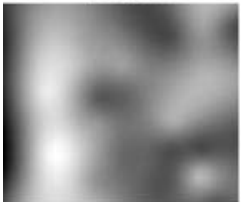

Mode 6

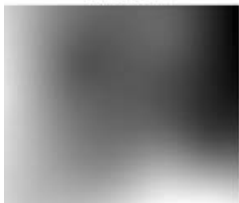

Mode 7

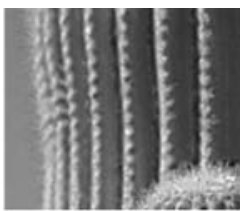

Residue of Mode 1

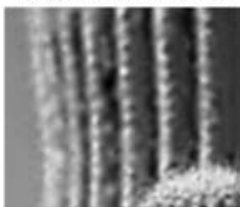

Residue of Mode 2

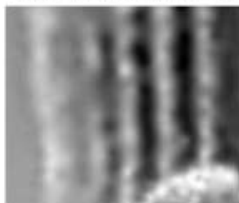

Residue of Mode 3

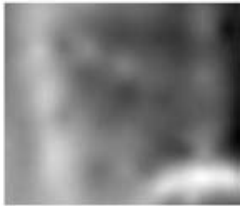

Residue of Mode 4

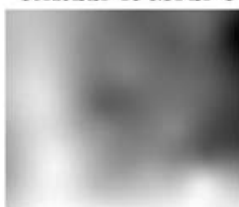

Residue of Mode 5

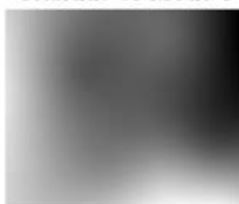

Residue of Mode 6

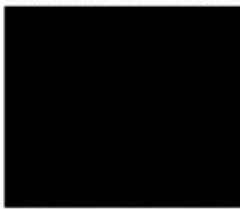

Residue of Mode 7

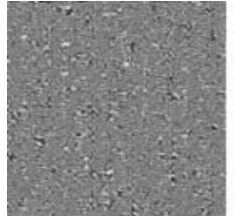

Mode 1

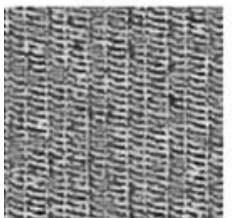

Mode 2

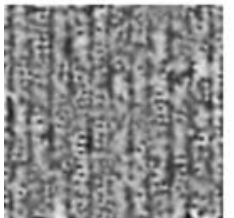

Mode 3

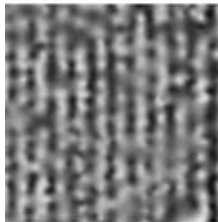

Mode 4

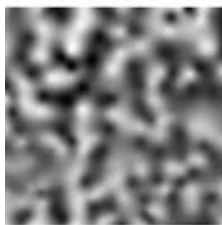

Mode 5

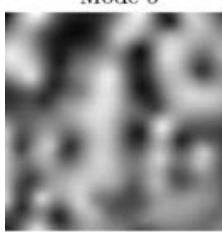

Mode 6

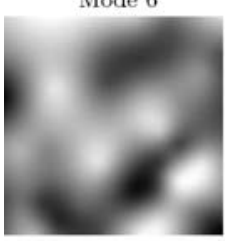

Mode 7

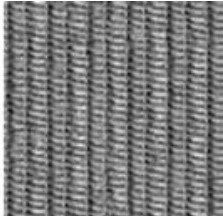

Residue of Mode 1

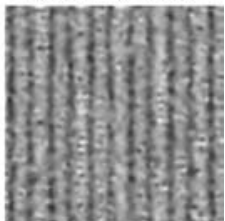

Residue of Mode 2

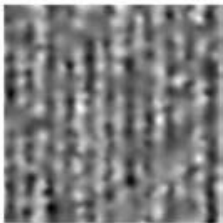

Residue of Mode 3

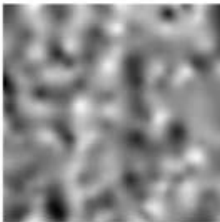

Residue of Mode 4

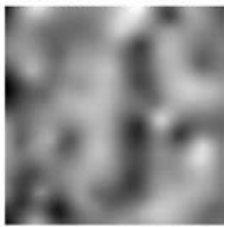

Residue of Mode 5

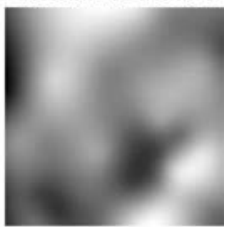

Residue of Mode 6

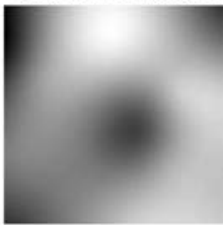

Residue of Mode 7
Fig. 13. BEMD of cactus image (Fig. 5) by TPS from extrema obtained by morphological reconstruction.
Fig. 14. BEMD of fabric image (Fig. 9) by TPS from extrema obtained by morphological reconstruction. 


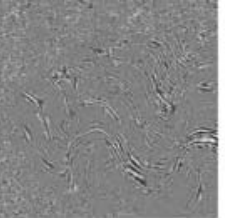

Mode 1

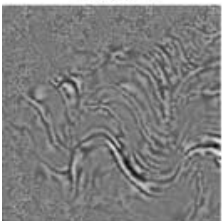

Mode 2

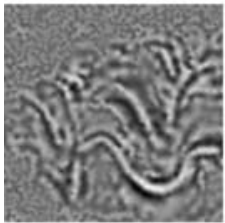

Mode 3

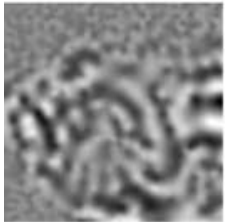

Mode 4

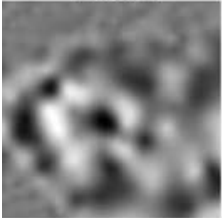

Mode 5

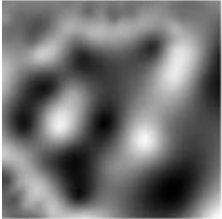

Mode 6

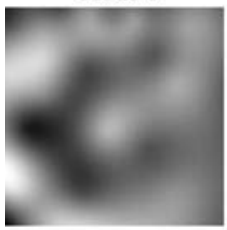

Mode 7

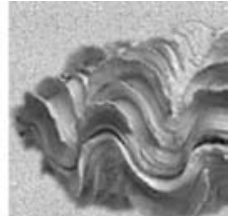

Residue of Mode 1

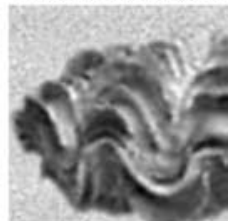

Residue of Mode 2

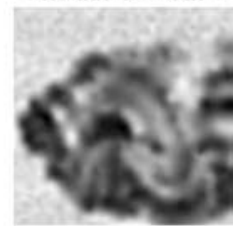

Residue of Mode 3

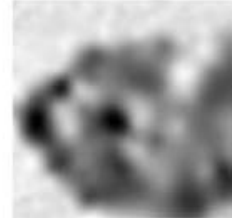

Residue of Mode 4

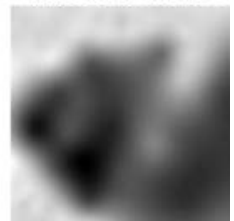

Residue of Mode 5

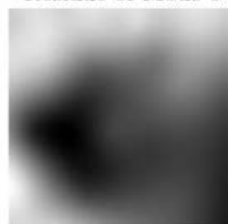

Residue of Mode 6

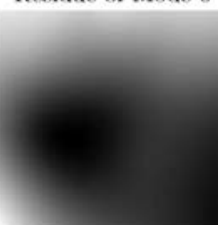

Residue of Mode 7

Fig. 15. BEMD of wavy image (Fig. 10) by TPS from extrema obtained by morphological reconstruction.

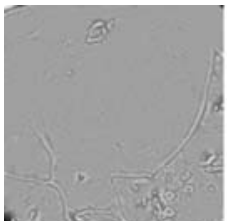

Mode 1

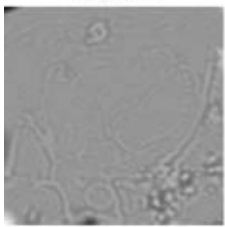

Mode 2

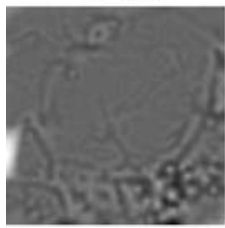

Mode 3

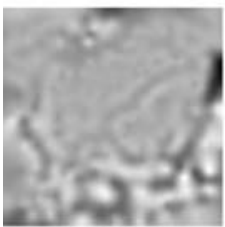

Mode 4

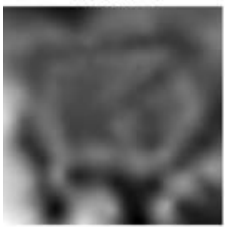

Mode 5

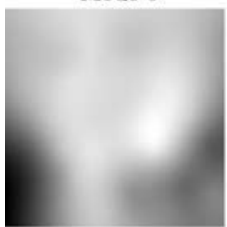

Mode 6

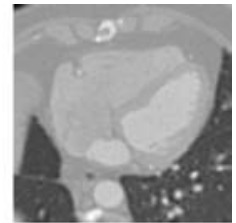

Residue of Mode 1

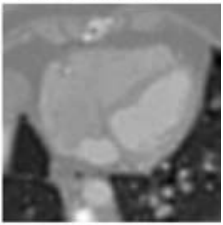

Residue of Mode 2

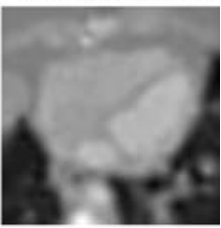

Residue of Mode 3

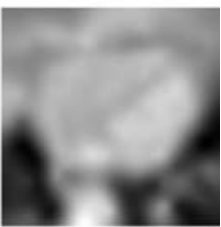

Residue of Mode 4

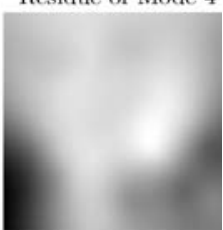

Residue of Mode 5

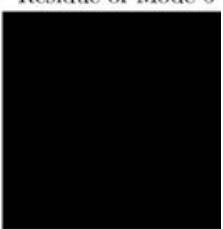

Residue of Mode 6
Fig. 16. BEMD of cardiac CT (Fig. 6). 


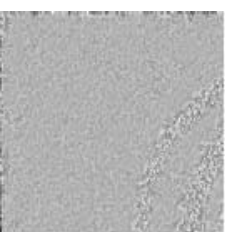

Mode 1

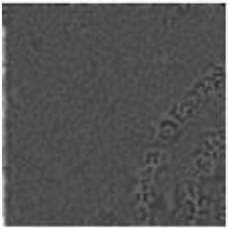

Mode 2

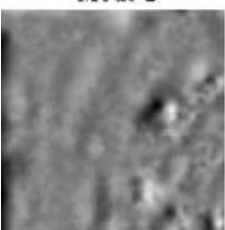

Mode 3

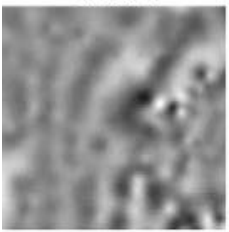

Mode 4

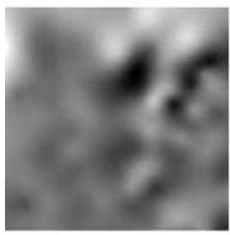

Mode 5

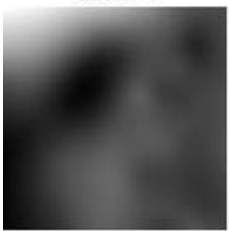

Mode 6

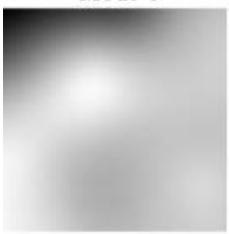

Mode 7

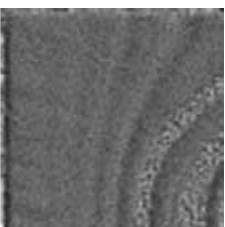

Residue of Mode 1

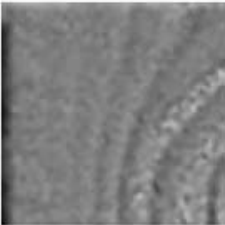

Residue of Mode 2

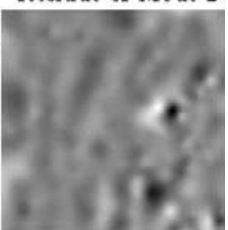

Residue of Mode 3

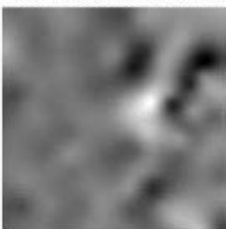

Residue of Mode 4

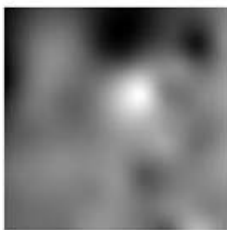

Residue of Mode 5

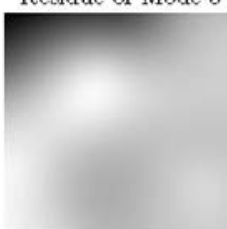

Residue of Mode 6

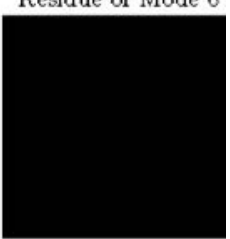

R.esidue of Mode 7

Fig. 17. BEMD of landshaft image (Fig. 7) by TPS from strict extrema.
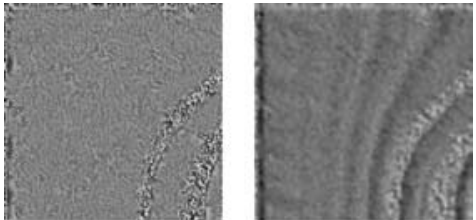

Mode 1

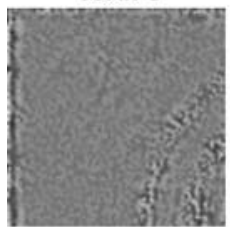

Mode 2

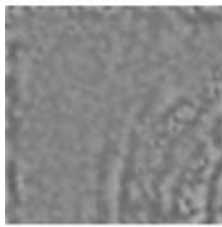

Mode 3

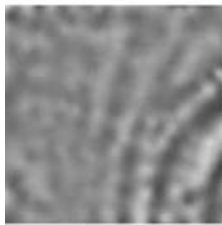

Mode 4

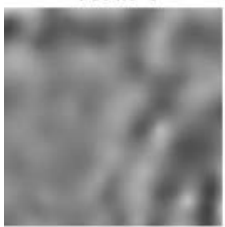

Mode 5

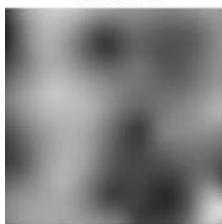

Mode 6

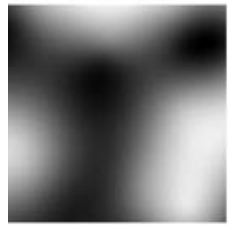

Mode 7

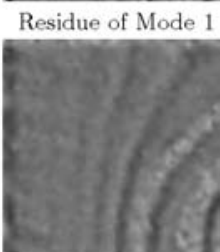

Residue of Mode 2

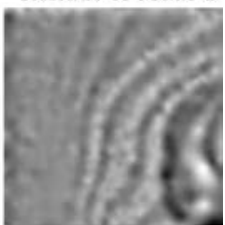

Residue of Mode 3

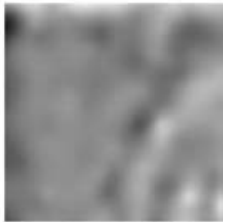

Residue of Mode 4

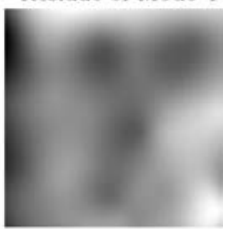

Residue of Mode 5

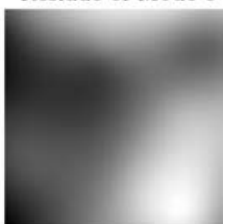

Residue of Mode 6

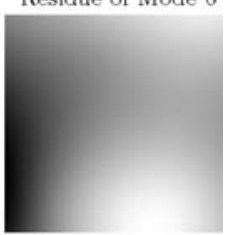

Residue of Mode 7

Fig. 18. BEMD of landshaft (Fig. 7) image by TPS from extrema obtained by morphological reconstruction. 


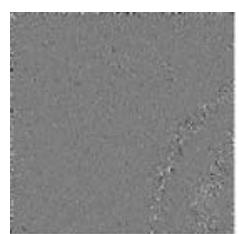

Mode 1

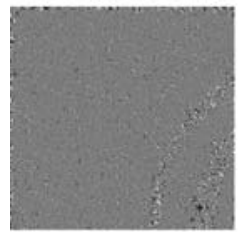

Mode 2

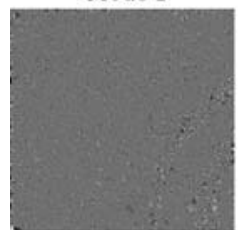

Mode 3

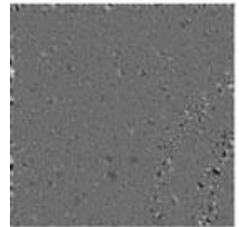

Mode 4

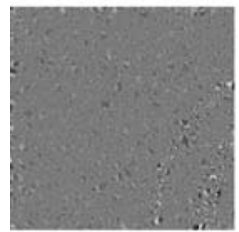

Mode 5

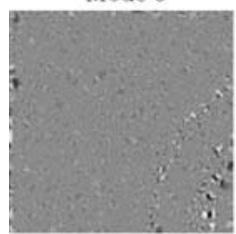

Mode 6

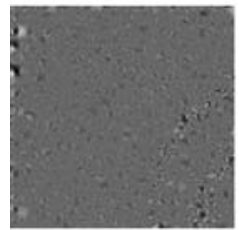

Mode 7

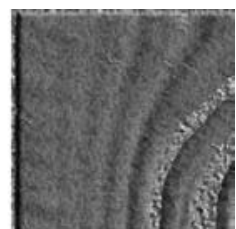

Residue of Mode 1

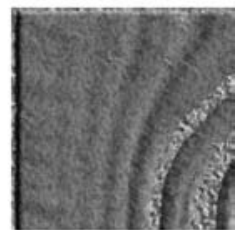

Residue of Mode 2

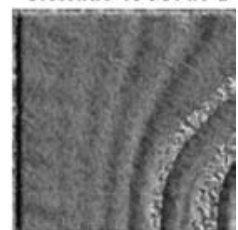

Residue of Mode 3

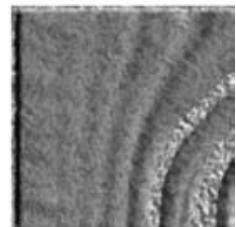

Residue of Mode 4

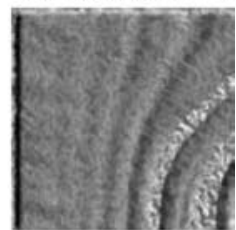

Residue of Mode 5

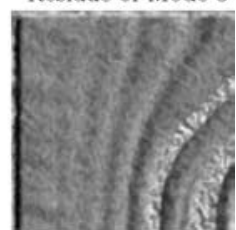

Residue of Mode 6

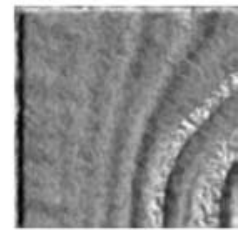

Residue of Mode 7

Fig. 19. BEMD of landshaft image (Fig. 7) by TPS from extrema obtained by watershed.
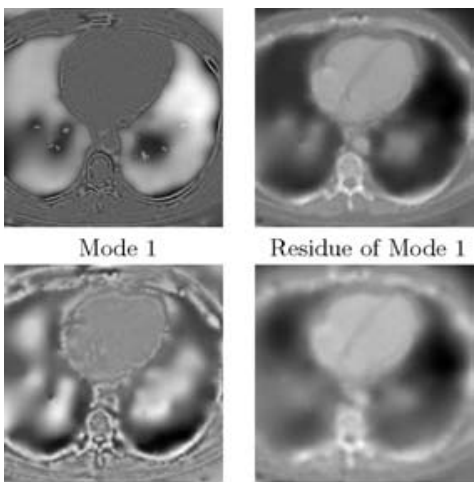

Mode 2

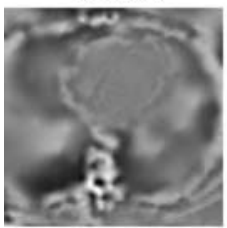

Mode 3

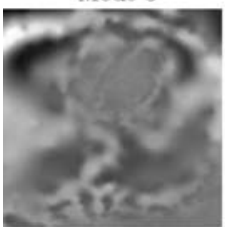

Mode 4

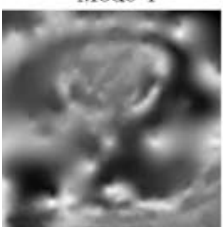

Mode 5

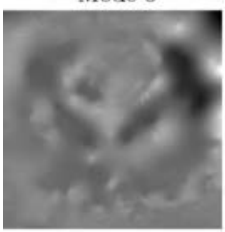

Mode 6

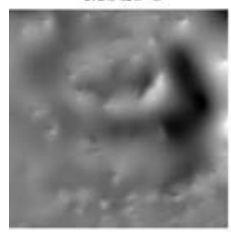

Mode 7

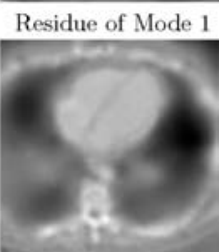

Residue of Mode 2

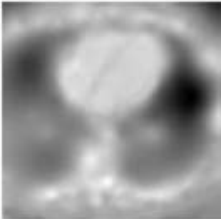

Residue of Mode 3

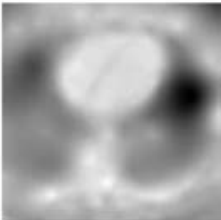

Residue of Mode 4

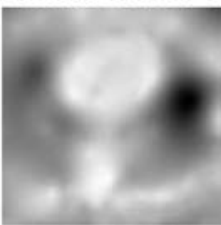

Residue of Mode 5

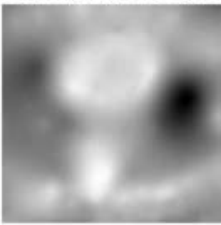

Residue of Mode 6

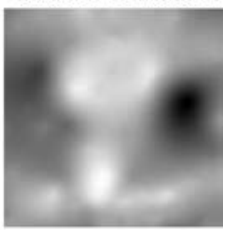

Residue of Mode 7

Fig. 20. BEMD of heart image (Fig. 8) by TPS from extrema obtained by morphological reconstruction (with precision $=0.00000003$ ). 


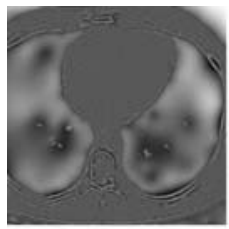

Mode 1

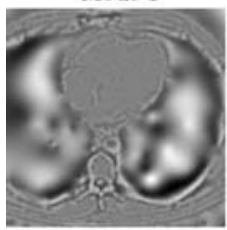

Mode 2

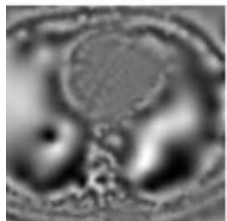

Mode 3

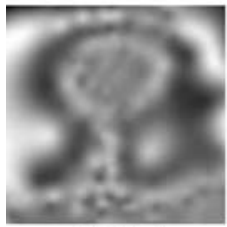

Mode 4

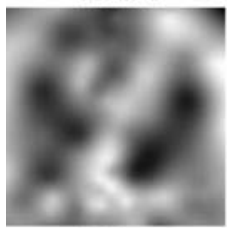

Mode 5

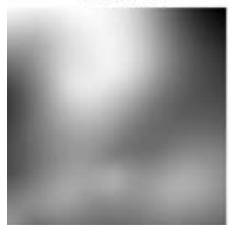

Mode 6

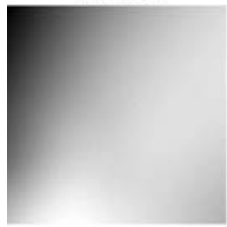

Mode 7

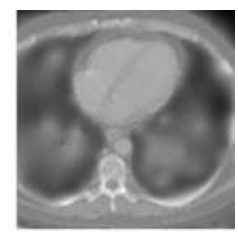

Residue of Mode 1

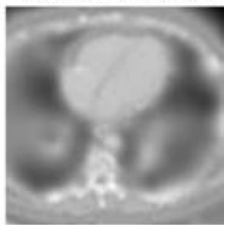

Residue of Mode 2

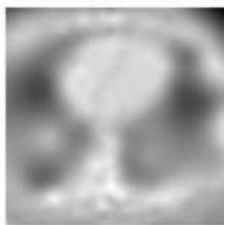

Residue of Mode 3

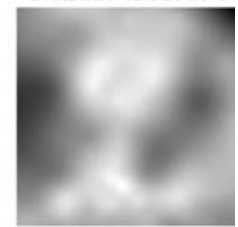

Residue of Mode 4

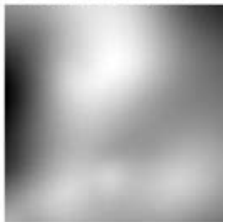

Residue of Mode 5

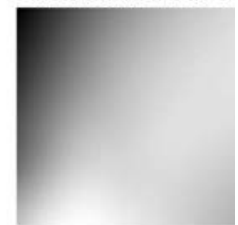

Residue of Mode 6

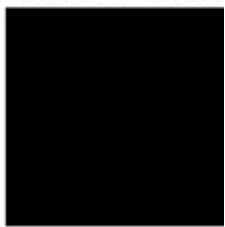

Residue of Mode 7

Fig. 21. BEMD of heart image (Fig. 8) by TPS from extrema obtained by morphological reconstruction (with precision $=0.00003$ ).
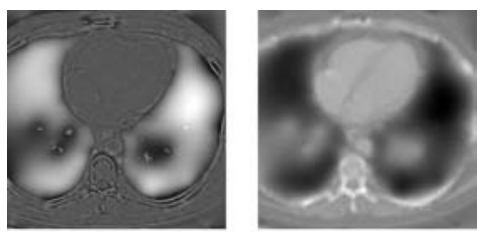

Mode 1

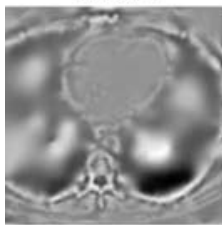

Mode 2

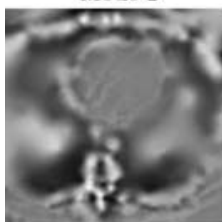

Mode 3

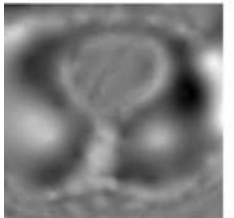

Mode 4

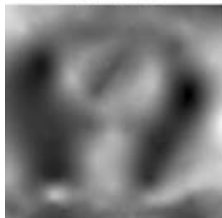

Mode 5

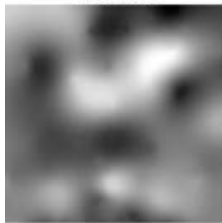

Mode 6

Residue of Mode 1

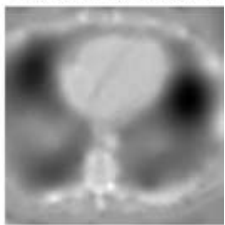

Residue of Mode 2

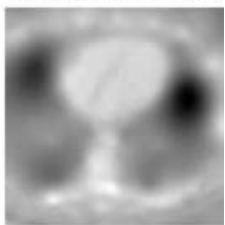

Residue of Mode 3

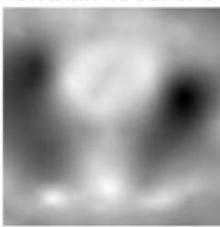

Residue of Mode 4

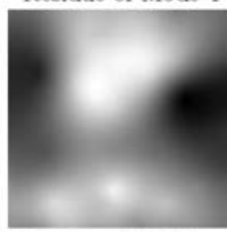

Residue of Mode 5

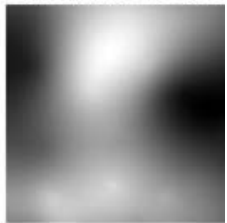

Residue of Mode 6

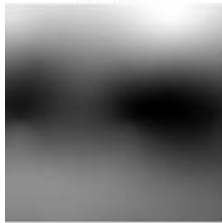

Mode 7

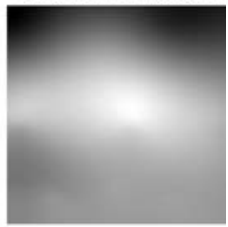

Residue of Mode 7

Fig. 22. BEMD of heart image (Fig. 8) by TPS from extrema obtained by morphological reconstruction (with precision $=0.03$ ). 
BEMD from three precision (0.00000003, 0.00003, and 0.03) from extrema obtained by morphological reconstruction.

\subsubsection{Zero-crossings}

We detect the zero-crossings of each modes and suggest a multiscale segmentation from these zero-crossings (such as proposed in Ref. 74). These zero-crossings can correspond to structures to seek. A multiscale segmentation approach perhaps suggested similar to that proposed by Marr-Hildreth ${ }^{70}$ using the zero-crossings of the Laplacian of Gaussian (Figs. 23-28).

\section{Fractal Synthesis by EMD}

We present here our work which was published in Ref. 23. We reported here that EMD method might offer a new way to synthesis fractional processes in $1 \mathrm{D}$ and $2 \mathrm{D}$ space.

\subsection{Fractal analysis}

The estimation of the fractal dimension, or of the Hurst exponent of an fBm realization has proven to be an important problem both for signal and image analysis. The fBm model has been successfully used in texture analysis and synthesis, landscape modeling and speech segmentation. Especially, in computer vision, Brownian texture is a widely used Gaussian process with a variety of applications in image analysis, e.g., in physics, medical, and fractal imaging.

\subsubsection{Analysis of fractional Brownian motion}

Fractional Brownian motion (hereafter $\mathrm{fBm}$ ) is a continuous-time random process proposed by Mandelbrot and Van Ness. ${ }^{69}$ Basically, it consists in a fractional integration of a white Gaussian process and is therefore a generalization of Brownian motion, which consists simply in a standard integration of a white Gaussian process.

In fact, $\mathrm{fBm}$ is the only known correlation model that satisfies Wornells definition of $1 / f$ processes. ${ }^{95}$ One characterizing feature of $\mathrm{fBm}$ is its statistical self-similar property, ${ }^{69}$ which means that the variance of increments of a process obeys a hyperbolic scaling law so that the statistical properties of the process at any two scales are the same within a scaling constant. The exponent of the hyperbolic law deals with the Hurst parameter $H$ that quantifies the persistence of an fBm realization. In fractal theory, Hurst exponent is related to the fractal dimension in a simple manner. ${ }^{30}$

Traditional analysis techniques are based on some type of regression analysis to measure the hyperbolic progression of the average size of increments at varying scales, the progression of power versus frequency, or the progression of the variance of the wavelet coefficients at different scales. ${ }^{38,43,80}$ A maximum likelihood fractal and fGn estimators were proposed in Refs. 24 and 67. Recently, an estimator 


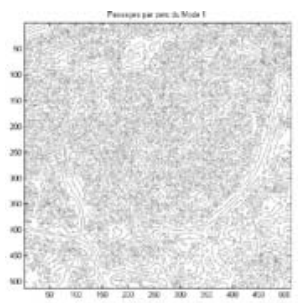

Zero crossings of Mode 1

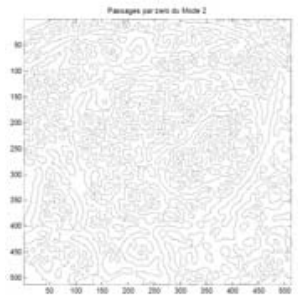

Zero crossings of Mode 2

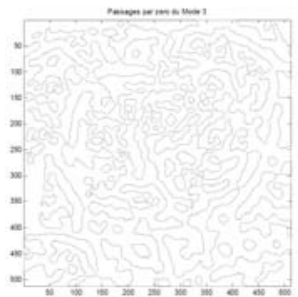

Zero crossings of Mode 3

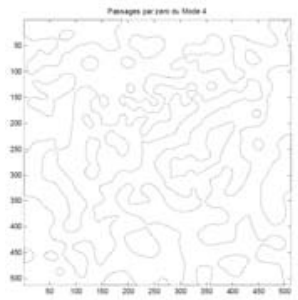

Zero crossings of Mode 4

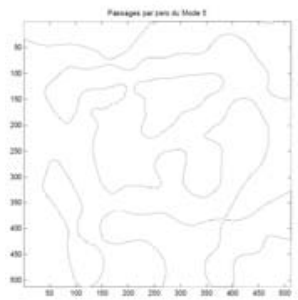

Zero crossings of Mode 5

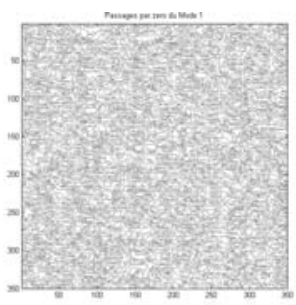

Zero crossings of Mode 1

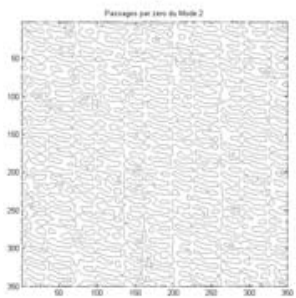

Zero crossings of Mode 2

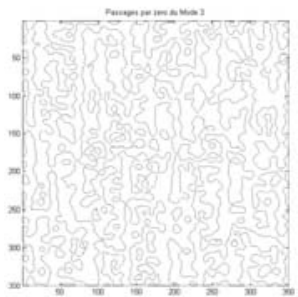

Zero crossings of Mode 3

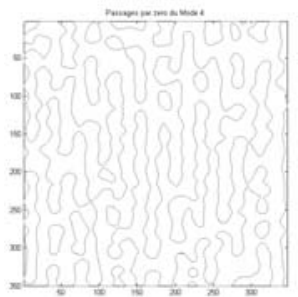

Zero crossings of Mode 4

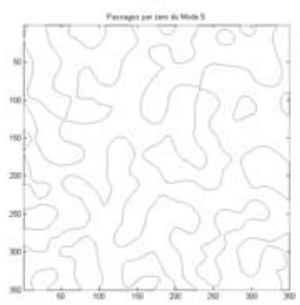

Zero crossings of Mode 5

Fig. 23. Zero-crossings of IMF (Fig. 16) Fig. 24. Zero-crossings of IMF (Fig. 14) obtained by BEMD of cardiac CT (Fig. 6). obtained by BEMD of fabric image (Fig. 9). 


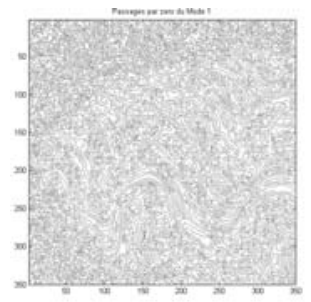

Zero crossings of Mode 1

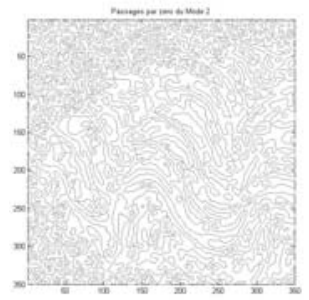

Zero crossings of Mode 2

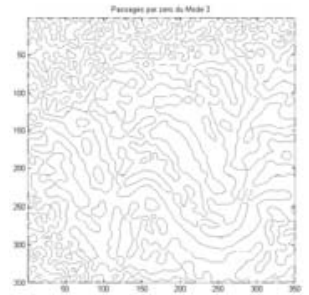

Zero crossings of Mode 3

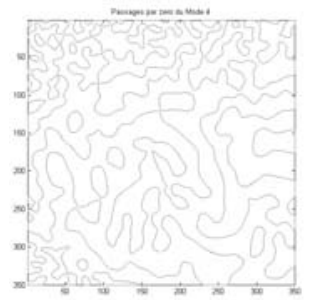

Zero crossings of Mode 4

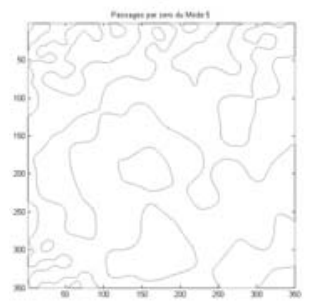

Zero crossings of Mode 5

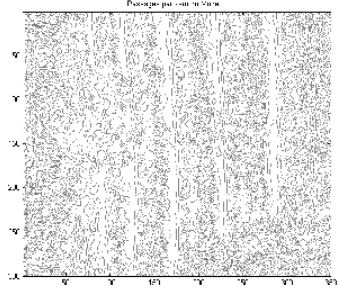

Zero crossings of Mode 1

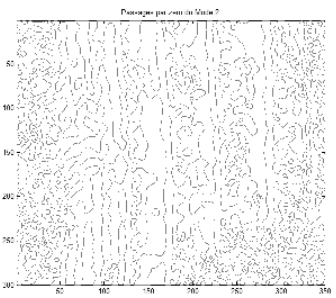

Zero crossings of Mode 2

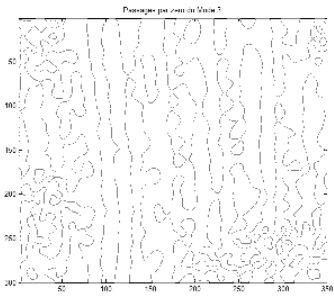

Zero crossings of Mode 3

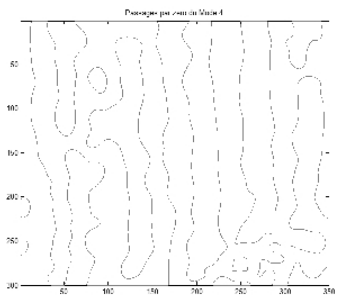

Zero crossings of Mode 4

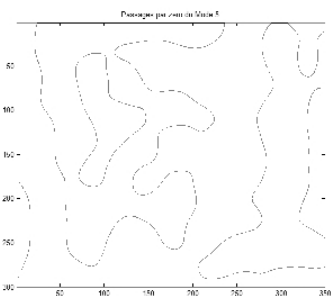

Zero crossings of Mode 5

Fig. 25. Zero-crossings of IMF (Fig. 15) Fig. 26. Zero-crossings of IMF (Fig. 13) obtained by BEMD of wavy image (Fig. 10). obtained by BEMD of cactus image (Fig. 5). 


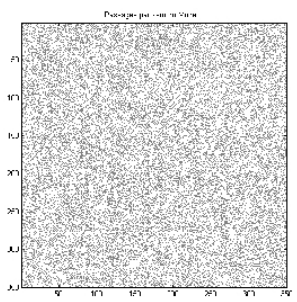

Zero crossings of Mode 1

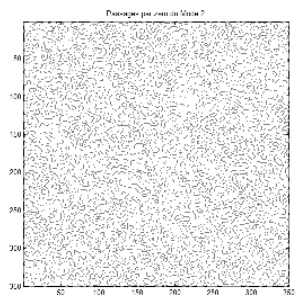

Zero crossings of Mode 2

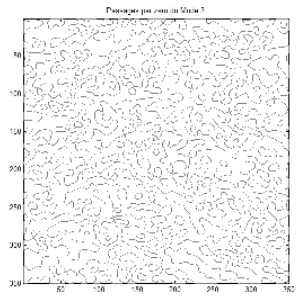

Zero crossings of Mode 3

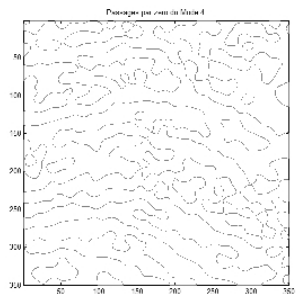

Zero crossings of Mode 4

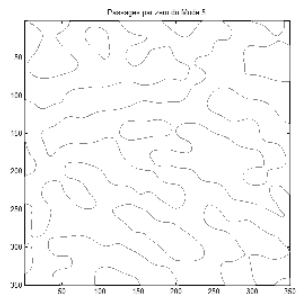

Zero crossings of Mode 5

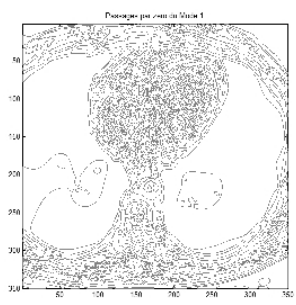

Zero crossings of Mode 1

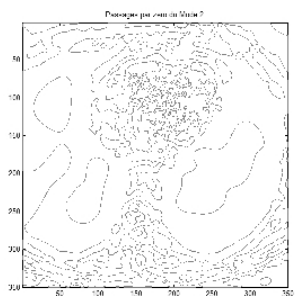

Zero crossings of Mode 2

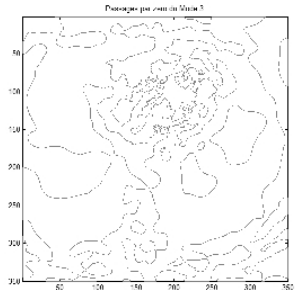

Zero crossings of Mode 3

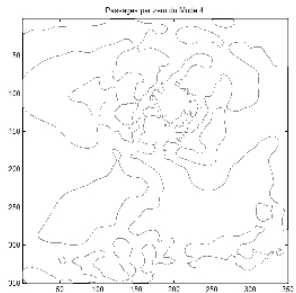

Zero crossings of Mode 4

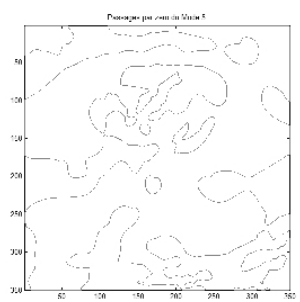

Zero crossings of Mode 5

Fig. 27. Zero-crossings of IMF (Fig. 12) Fig. 28. Zero-crossings of IMF (Fig. 20) obtained by BEMD of abbey texture (Fig. 4). obtained by BEMD of cardiac CT (Fig. 8). 
for noisy $\mathrm{fBm}$ measurements that takes advantage of the decorrelation effects of orthogonal wavelets was published in Refs. 1 and 96 .

More recently, EMD application to some broadband processes have been shown to spontaneously achieve wavelet-like decompositions. ${ }^{39,40}$

\subsubsection{Dyadic filter-bank structure of EMD}

The experimental spectral analysis and statistical characterization of the obtained IMFs reveal an equivalent filter-bank structure which shares most properties of a wavelet decomposition in the same context, ${ }^{1}$ in terms of self-similarity, quasidecorrelation and variance progression. ${ }^{37,39,40,99}$ Furthermore, the spontaneous adaptation of EMD to "natural" dyadic scales is shown, rationalizing the method as an alternative way for estimating the fractional-Gaussian-noise Hurst exponent. ${ }^{39,40}$

The authors ${ }^{37,39}$ estimate a power spectrum for each IMF of the decomposition. To renormalize according to the power spectra in a coupled way these modes, either in time, or in frequency provides a single curve. Moreover, the number of these zero-crossings (or extrema) $z_{H}(j)$ is a decreasing exponential function of the mode number $j$ : $z_{H}(j)=\rho_{H}^{-j}$, with $\rho_{H}$ very close to 2 . The spectra of all modes (for indices 2 to last) look quite the same: a band-pass filter. The spectrum of the first mode corresponds to the characteristics of a high-pass filter. The logarithm of the maximum amplitude of modes 2 to last (for EMD of impulse) varies linearly according to the index of the modes. ${ }^{37,39}$

\subsubsection{EMD-based characterization of stationary processes}

Fractional Gaussian noise (fGn) can be viewed as the increment process of fractional Brownian motion (fBm). ${ }^{69} \mathrm{An} \mathrm{fBm}$ process, $B_{H}$, is a so-called $H$-sssi Gaussian process, meaning that it is self-similar with index $H \in(0,1)$ and has stationary (discrete-time) increments $s_{H}[j] \cong B_{H}[j+1]-B_{H}[j]$ with autocovariance function $\gamma_{H}[j]=E\left\{s_{H}[n] s_{H}[n+j]\right\}^{29}$ given by

$$
\gamma_{H}[j]=\frac{\sigma^{2}}{2}\left(|j-1|^{2 H}-2|j|^{2 H}+|j+1|^{2 H}\right) .
$$

White noise increments correspond to the special case of fGn realization for which $H=1 / 2$. It is shown in Refs. 40, 83, 99 that in the case of fractional Gaussian noise, EMD can be interpreted as a filter bank of overlapping band-pass filters for modes of ranks $j \leq 2$

$$
\operatorname{Var}\left\{C_{H}^{\langle j\rangle}(t)\right\} \propto \rho_{H}^{j(2 H-2)},
$$

where $C_{H}^{\langle j\rangle}(t)$ is the $j$ th IMF of process $s_{H}^{(t)}$. The dyadic nature of the EMD on fractional processes implies $\rho_{H} \approx 2$ for all $H .{ }^{40}$ Hence, it becomes possible to access to the Hurst exponent via the variance progression across IMFs

$$
\operatorname{Var}\left\{C_{H}^{\langle j\rangle}(t)\right\} \propto 2^{j(2 H-2)} .
$$


In Sec. 3.2, we used this remarkable property to propose a new algorithm for stationary processes simulation.

\subsection{Fractal synthesis by EMD}

There exist algorithms for simulating general fractional processes with a given autocovariance function. These algorithms can be used for generating 1D signals or texture images. Textures and natural data are often modeled by the increments of the sampled fBm, known as (discrete) fractional Gaussian noise (fGn). Therefore, fBm and fGn, or simply fractal models, have been successfully applied to texture analysis and synthesis ${ }^{19,54,80,89}$ and terrain modeling. ${ }^{55,79,98}$ For a general point of view, there exist several methods for generating long-range dependent processes, such as fBm or fGn. Two classes of methods can be defined;

- Cholesky decomposition methods based on fGn covariance matrix, and

- spectral, ${ }^{68}$ wavelets $^{38}$ or autoregressive models ${ }^{90}$ methods.

See Ref. 5 for a survey about these methods.

\subsubsection{Proposed fractional processes synthesis method}

Decomposition of white noise: An extended analysis of characterization of ordinary Gaussian noise based on EMD method is presented in Refs. 9. Following the results presented in Refs. 40 and 99 and relation, we known that the decomposition of ordinary Gaussian noise, $H=1 / 2$, by use of EMD ends up with IMFs with variance progression $\operatorname{Var}\left\{C_{1 / 2}^{\langle j\rangle}(t)\right\} \propto \rho_{H}^{-j}$. Hence, it is possible to generate fractional Gaussian noise by summing all IMFs with appropriately weighting factors. We call this $H$-dependent EMD reconstruction.

$H$-dependent EMD reconstruction: Based on EMD of White noise, the following algorithm generates fractional processes with Hurst exponent $H$, see also Ref. 23:

- Initialization. Generate ordinary Gaussian noise, $\xi(t)$, for which $H=1 / 2$.

- Decomposition. Decompose $\xi(t)$ with EMD method to generate the $J$ IMFs $C_{1 / 2}^{\langle j\rangle}(t), j=1, \ldots, J$ of the representation of $\xi(t)$.

- Reconstruction. Weight each IMF of rank $j$ by factor $2^{j(\beta)} / \sigma\langle j\rangle$, where $H$ is the desired new Hurst exponent, and reconstruct new fractional process $\xi_{H}(t)$ by the following EMD reconstruction, where $\sigma^{\langle j\rangle}$ stands for standard deviation of the $j$ th IMF:

$$
\xi_{h}(t)=\sum_{j=1}^{J} \frac{2^{j(\beta-1)}}{\sigma^{\langle j\rangle}} C_{1 / 2}^{\langle j\rangle}(t)
$$


with

$$
\beta= \begin{cases}H & \text { for an fGn } \\ H+1 & \text { for an fBm. }\end{cases}
$$

Parameter $\beta$ is related with Hurst exponent $H$ as in Eq. (15) for, respectively, fGn or fBm processes synthesis. The $H$-dependent EMD reconstruction, Eq. (14), not holds the trend $r^{\langle J\rangle}(t)$ of initial representation. In practice, this residual reflecting global trend is a negligible component of EMD of White noise. Alternatively, it can be considered as the $(J+1)$ th IMF of the EMD, and can be integrated in Eq. (14).

Figure 29 shows the theoretical autocorrelation and the real autocorrelation of fGn simulations (obtained by EMD) according to different values of Hurst exponent $H=0.1,0.2,0.8,0.9$. Fractional processes (5000) are generated from the White noise realizations (5000) of data length $N=1000$. We show a centered window on peak of autocorrelation -20 to +20 . We show in gray the theoretical autocorrelation and black the autocorrelation of fBm obtained by EMD.

\subsubsection{Texture synthesis}

IMFs variance progression in the $2 D$ case: In the spirit of the previous work on characterization of EMD method in $1 \mathrm{D}$ case, ${ }^{40}$ we have found that the number of zero-crossings, $z_{H}$, for each bidimensional IMF obtained from the decomposition of fractional Gaussian noise images are related to rank $j$ by the power law:

$$
z_{H}=\rho_{H}^{-j}
$$

with $\rho_{H} \approx 2$ for all $H \in(0,1)$, which is a mark of dyadic nature of BEMD as in $1 \mathrm{D}$ case. Therefore, we can write the variance progression across BIMFs as

$$
\operatorname{Var}\left\{C_{H}^{\langle j\rangle}(x, y)\right\} \propto 2^{-j(2 \beta-2)},
$$

where parameter $\beta$ is related to Hurst exponent $H$ as in Eq. (15).

To extract the $2 \mathrm{D}$-IMF during the $2 \mathrm{D}$-sifting process $^{74}$ we have used morphological operators to detect the image extrema and TPS to compute the surface interpolation (Fig. 1). We present in (Fig. 30) 2D-IMF of impulse realizations (500 realizations) for six first IMF.

Figure 31 show average according to 16 directions of impulse response of BEMD.

Figure 32 shows the average and logarithm of average number of extrema by 2D-IMF.

Bidimensional fractional processes synthesis algorithm: With Eq. (17), the extension of $H$-dependent reconstruction, expressed by Eq. (14), in $2 \mathrm{D}$ case is immediately expressed by

$$
\xi_{H}(x, y)=\sum_{j=1}^{J} \frac{2^{j(\beta-1)}}{\sigma^{\langle j\rangle}} C_{1 / 2}^{\langle j\rangle}(x, y)
$$




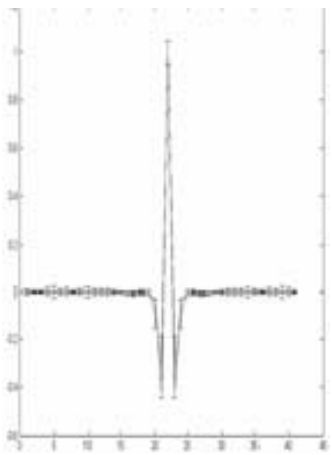

(a) $H=0.1$

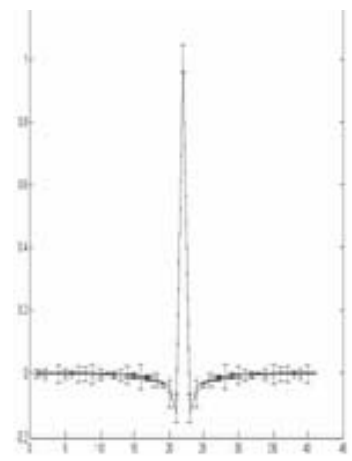

(d) $H=0.4$

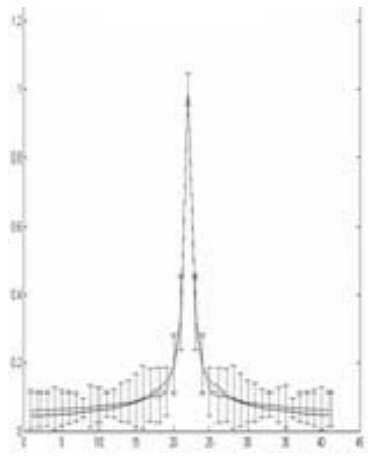

(g) $H=0.7$

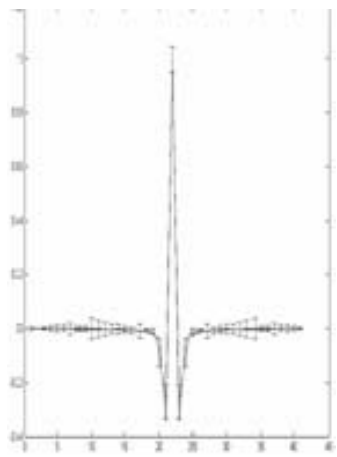

(b) $H=0.2$

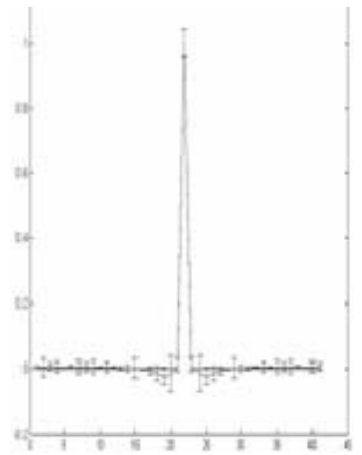

(e) $H=0.5$

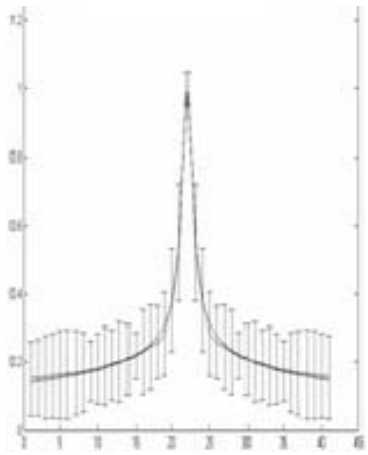

(h) $H=0.8$

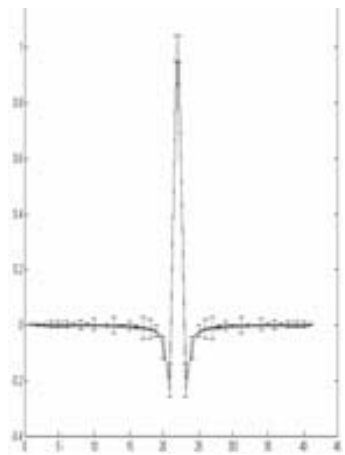

(c) $H=0.3$

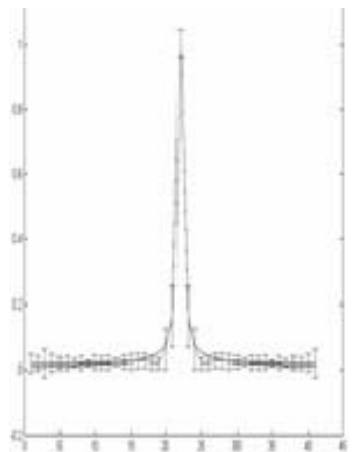

(f) $H=0.6$

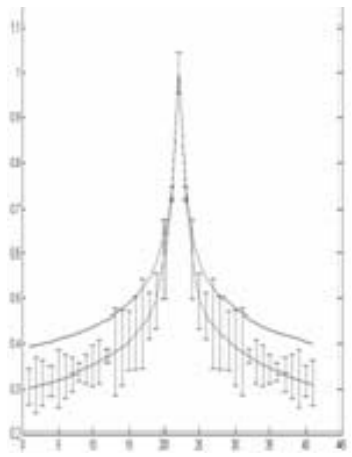

(i) $H=0.9$

Fig. 29. Theoretical and real autocorrelation of EMD-based simulation of fractional processes according to different values of Hurst exponent $H=0.1,0.2,0.8,0.9$. First published in Ref. 23.

where $\xi_{H}$ is the generated fGn or fBm 2D process depending on the choice of parameter $\beta$ as in Eq. (15).

Figure 33 shows different realizations of $\mathrm{fGn}$ and $\mathrm{fBm}$ texture images. The BEMD-based synthesis results depend on the choice of extrema definition in the 


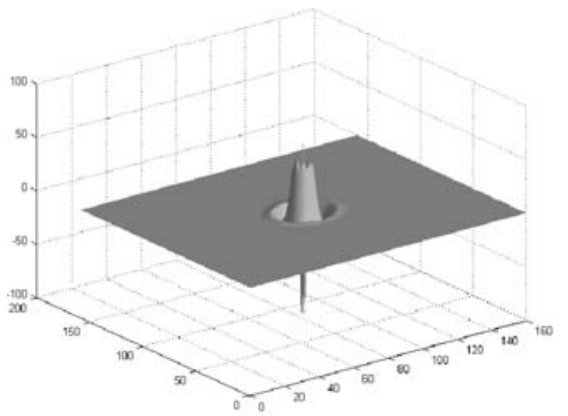

Mode 1

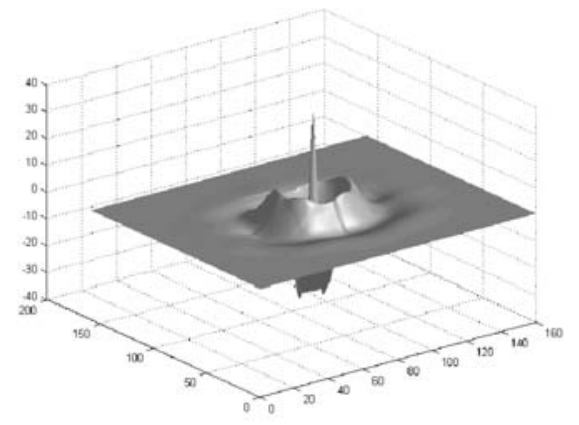

Mode 3

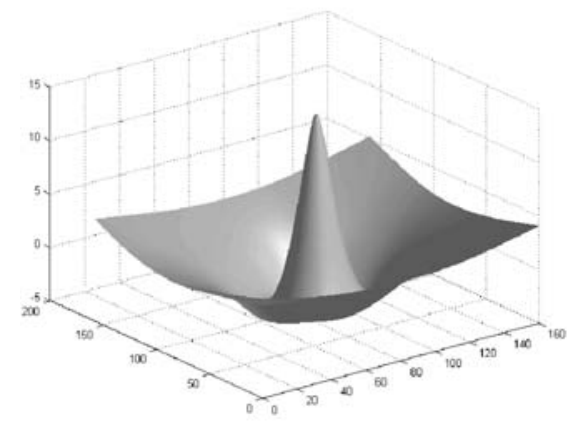

Mode 5

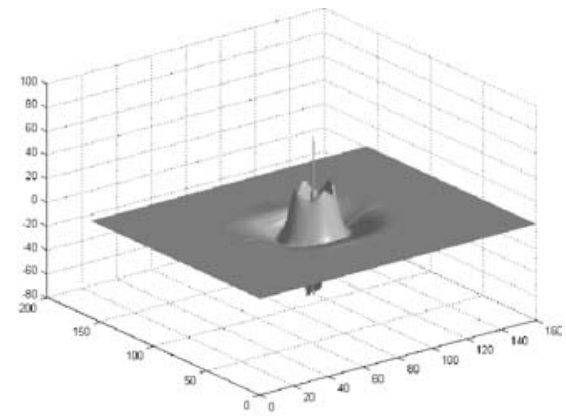

Mode 2

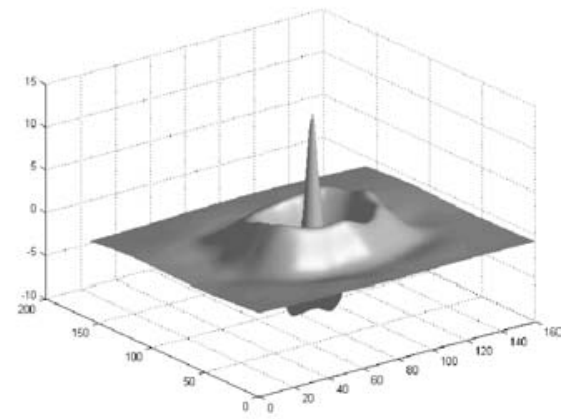

Mode 4

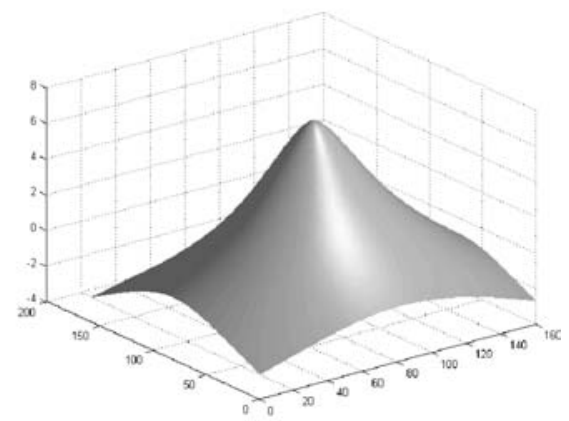

Fig. 30. Impulse response of BEMD. First published in Ref. 23. 


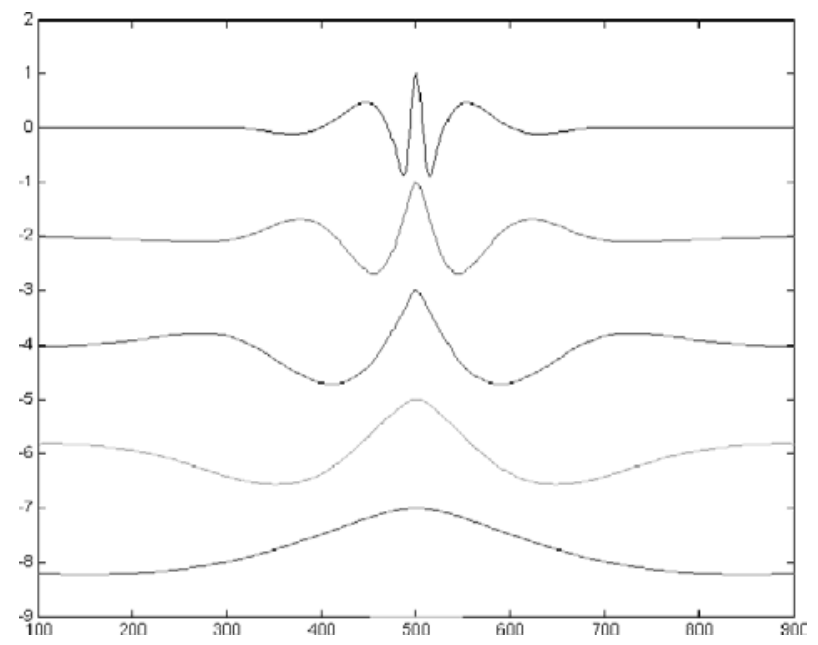

Fig. 31. Average according to 16 directions of impulse response of BEMD. First published in Ref. 23 .

sifting process, such as local extrema (Fig. 33(a)) or lines of extrema (Fig. 33(b)). In both cases, fractional processes are generated with different values of Hurst exponent $H=\{0.05,0.15,0.85,0.95\}$.

\section{Phase-Based Image Processing and BEMD}

We present here our work which was published in Ref. 76. The main contribution of our approach is to apply the Hilbert-Huang transform (which consists of two parts: (a) EMD, and (b) the Hilbert spectral analysis) to texture analysis.

We applied Hilbert-Huang transform ${ }^{51}$ (which consists of two parts: (a) EMD, and (b) Hilbert spectral analysis) to texture images for two reasons. ${ }^{74}$ The first reason is that EMD is a fully data-driven $\operatorname{method}^{77}$ and using no predetermined filter ${ }^{28}$ or wavelet functions. Indeed, we implemented a bidimensional EMD (BEMD). ${ }^{75}$ The second reason is that we can analyze locally and independently each 2D-IMF obtained by BEMD in the same way that through the Hilbert-Huang transform.

\subsection{The Riesz transform and the monogenic signal}

Such as the Hilbert-Huang transform in the case of one-dimensional signal analysis, ${ }^{52}$ we present an image analysis method by studying local properties of 2D-IMF obtained by BEMD. These local properties (amplitude, phase, isotropy, and orientation) are extracted from the monogenic signal of each 2D-IMF. The monogenic signal $^{31}$ is a $2 \mathrm{D}$-generalization of the analytic signal, where the Riesz transform replaces the Hilbert transform.

The estimation of the local phase and the local amplitude is an important step in many signal and image processing tasks. A second crucial task in many image 


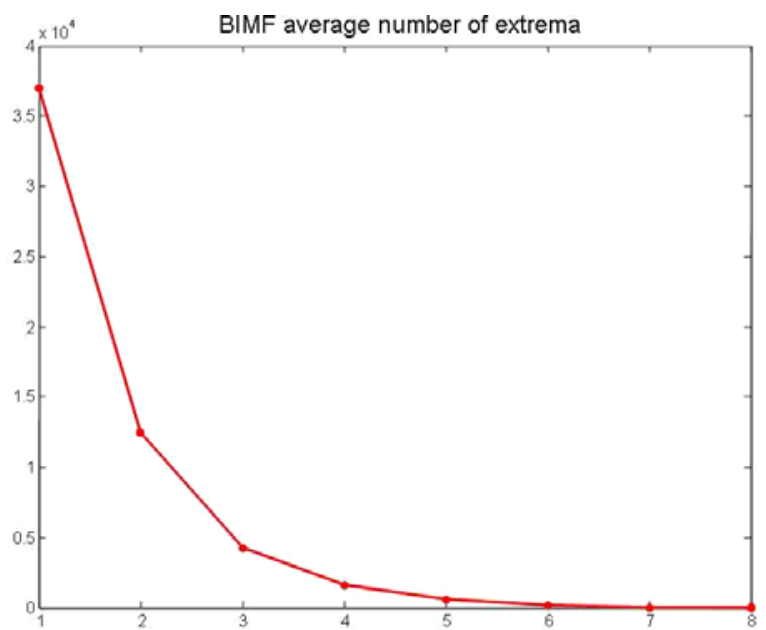

Average number of extrema of $2 D$-IMF.

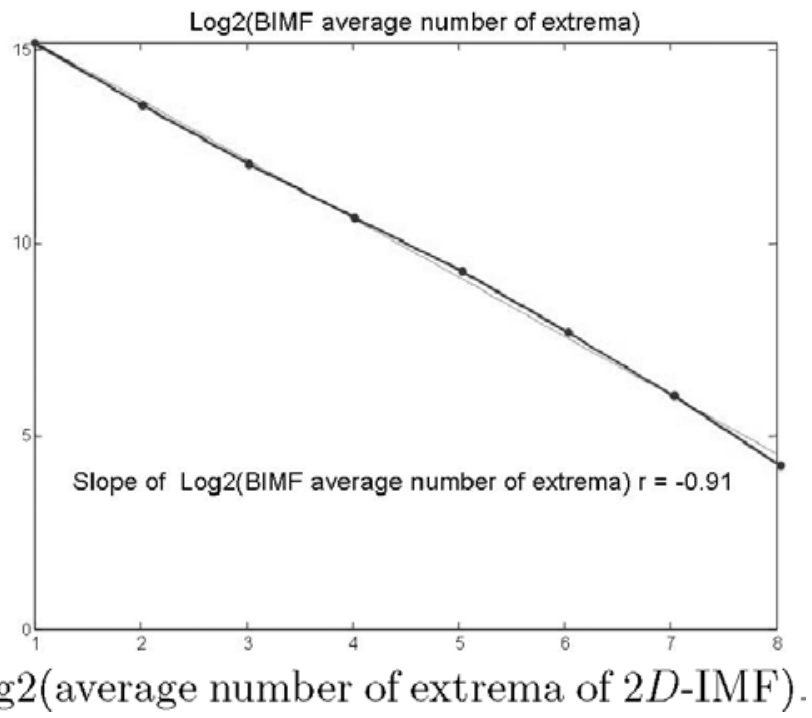

Fig. 32. Average number of extrema of 2D-IMF in the case of 2D-fractional Gaussian noise. First published in Ref. 23.

processing and more precisely in texture analysis is the estimation of the local orientation. Furthermore, the local frequency can be taken as a measure for local scale (i.e., the frequency band), ${ }^{53,78}$ structures such as lines and edges can be distinguished by the local phase ${ }^{45}$ the local amplitude and the local phase can be used for edge detection, ${ }^{58}$ and the local phase can be used to estimate the disparity of stereo images ${ }^{47}$ or the flow in image sequences. 

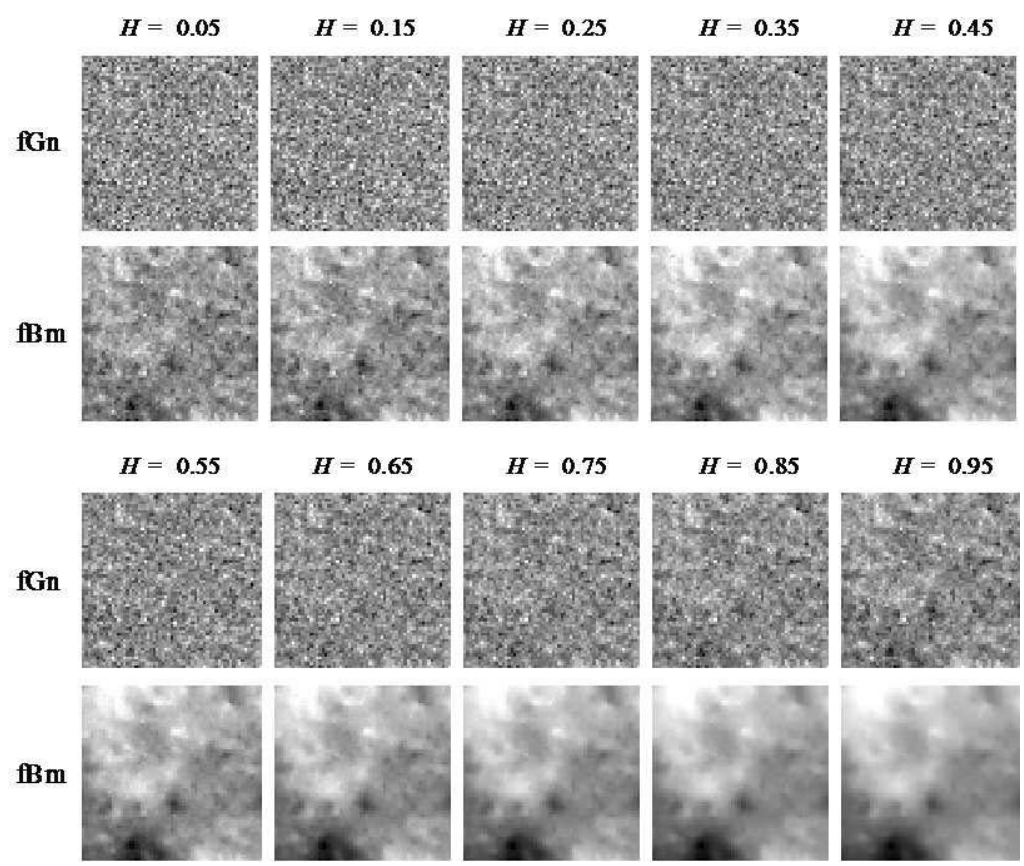

(a)
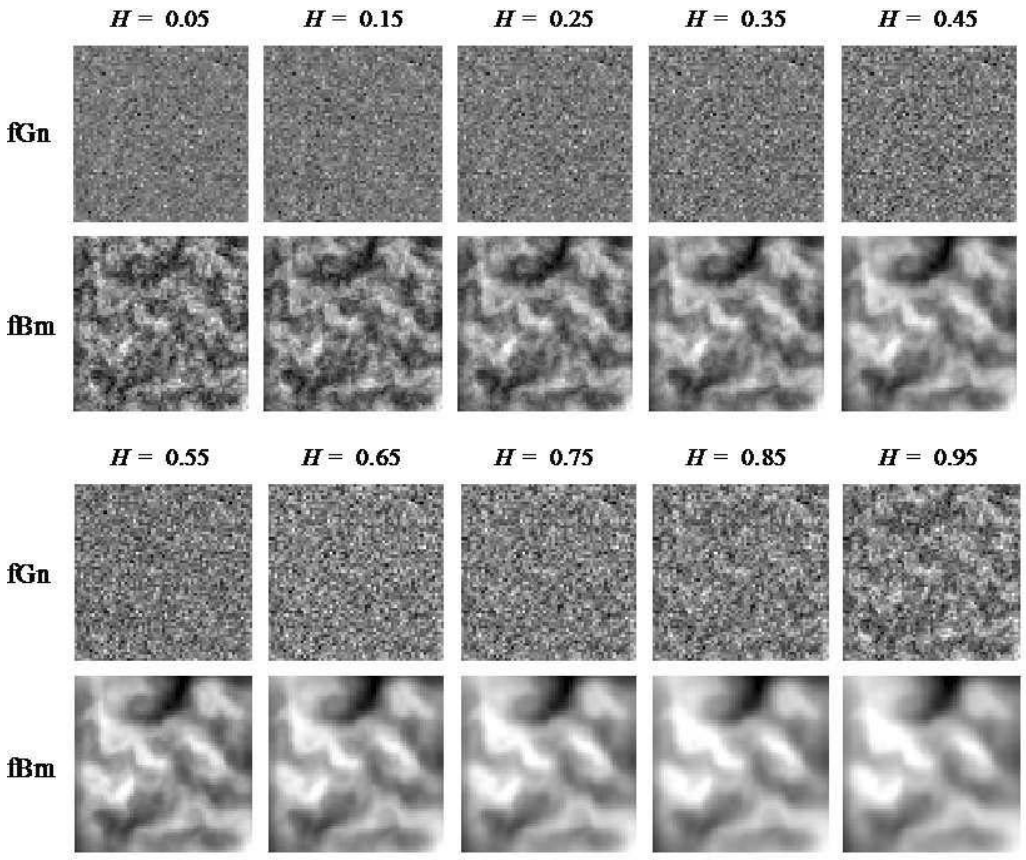

(b)

Fig. 33. EMD-based simulation of fractional processes according to different values of Hurst exponent $H=0.1,0.2,0.9$. First published in Ref. 23 . 
The methods used for the phase and amplitude estimation are based on the evaluation of the analytic signal of the input signal which involves the calculation of the signal's Hilbert transform.

\subsubsection{The Hilbert transform and analytic signal}

The analytic signal provides access to a real one-dimensional (1D) signal's local amplitude and phase. The complex signal is built from a real signal by adding its Hilbert transform — which is a phase-shifted version of the signal — as an imaginary part to the signal.

The Hilbert transform $f_{H i}$ of a real $1 \mathrm{D}$-signal $f$ is given by

$$
f_{H i}(x)=f(x) * \frac{1}{\pi x}
$$

where $*$ denotes convolution.

The complex signal $f_{A}$ of $f$ is the sum of the original signal and the phase-shifted signal, where the shifted signal is added as an imaginary part:

$$
f_{A}(x)=f(x)+i f_{H i}(x)=f(x) *\left(\delta(x)+\frac{i}{\pi x}\right) .
$$

The real part of $f_{A}$ is identical to the input signal, while the imaginary part is a $\left(-\frac{\pi}{2}\right.$ )-phase-shifted version (or the Hilbert transform $f_{H i}$ ) of $f$. In the frequency domain the Hilbert transform is defined by $F_{H i}(u)=-\frac{i u}{|u|} F(u)$, where $F$ and $F_{H i}$ are the Fourier transforms of $f$ and $f_{H i}$, respectively.

The analytic signal can be written as $f_{A}(x)=\left|f_{A}(x)\right| \exp (i \phi(x))$. Here $\left|f_{A}(x)\right|$ is called the local amplitude and $\phi(x)$ the local phase of $f$.

The complex signal can be used to separate the amplitude and the phase information of a given real 1D-signal. Since such a separation would be of helpful for multidimensional signals as well e.g., for feature extraction and classification in image processing, it is natural that there have been attempts to define the complex signal in $n D$.

\subsubsection{The Riesz transform and the monogenic signal}

In Ref. 33, the authors address the topics of scale-space and phase-based signal processing in common framework. The Riesz transform ${ }^{27}$ is a multidimensional generalization of the Hilbert transform. ${ }^{31}$ The combination of a $1 \mathrm{D}$-signal and its Hilbert transform is called the analytic signal. ${ }^{45}$ The analytic signal is the basis for all kinds of approaches which make use of the local phase. Similarly, the combination of a 2D-signal and its Riesz transform is called the monogenic signal. ${ }^{31}$ For a more detailed discussion on the monogenic signal, refer to Refs. 31 and 34 .

Indeed, monogenic signal is often identified as a local quantitative or qualitative measure of an image. Different approaches to an $n D$-analytic or complex signal 
have been proposed in the past:

- the total Hilbert transform, ${ }^{87}$

- the partial Hilbert transform, ${ }^{45}$

- the total complex signal, ${ }^{46}$

- the hypercomplex signal. ${ }^{16}$

The different approaches are unified by expressing all of them as combinations of the signal and its partial and total Hilbert transforms. It is known that the Riesz transform generalizes the Hilbert transform. ${ }^{86}$ Some of the scripts can be found in Refs. 34 and 84.

Phase-based image processing which have been proposed are:

- Edge detection by means of quadrature filters,

- Phase congruency is based on comparisons of the local phase at certain distinct scales, ${ }^{57,82}$

- Differential phase congruency. ${ }^{33}$

The main difference between approaches using the detection of local amplitude and phase congruency approaches is the behavior in $2 \mathrm{D}$-neighborhoods. ${ }^{33}$ This can also be verified in various edge-detection experiments, ${ }^{34}$ where the amplitude and based approaches blur the contour at corners and junctions.

\subsubsection{Structure MultiVector (SMV)}

The structure multivector is an approach for analyzing the local properties of two-dimensional signal (e.g., images). ${ }^{32}$ It combines the classical concepts of the structure tensor and the analytic signal in a new way. This has been made possible by using a representation in the algebra of quaternions. The resulting methods are linear and of low complexity. The filter-response includes local phase, local amplitude, and local orientation of intrinsically 1D neighborhoods in the signal. The authors of Ref. 32 develop a 2D-analytic signal for intrinsically $1 \mathrm{D}$-signals (in contrast to $2 \mathrm{D}$-analytic signal ${ }^{15}$ which is designed for intrinsically 2D-signals), which include three properties: local amplitude, local phase, and local orientation.

As already the name induces, the structure multivector is closely related to the structure tensor. The structure tensor as defined in Ref. 88 includes the following information (in fact, only the orientation vector is considered):

- amplitude: measurement for the existence of local structure,

- orientation of the local structure.

The structure multivector ${ }^{32}$ consists of three independent components (local phase, local orientation, and local amplitude) and its codes three properties. Consequently, there is no additional information possible. The function which computes the 
structure multivector uses a multiscale approach, because the shift of the Gaussian bandpass is coupled with the variance as for the Gabor wavelets.

Local phase and local amplitude are inherent features of scale-space theory. ${ }^{33}$ The ideas of local phase and multiscale representation are combined to recognize local structures and to measure local features. We used SMV to study local properties of each 2D-IMF.

\subsection{Phase-based image processing and BEMD}

Local properties extracted from the monogenic signal obtained by the structure multivector $^{32}$ of each 2D-IMF are the following ones: amplitude, phase, isotropy, directionality.

We show in Figs. 14 and 15 local features (major amplitude, minor amplitude, major phase, minor phase, isotropy and orientation) extracted by SMV of first 2D-IMF of Figs. 5(b) and 15(a).

These extracted local features have direct semantic interpretation. Indeed, we can analyze independently each one of these extracted local features and propose a new segmentation method not from the original image but from the local features of one or more 2D-IMF obtained by BEMD. For example, we can detect uniform areas in the image of major amplitude or orientation of first 2D-IMF.

Extracted by SMV of IMF (Fig. 20) obtained by BEMD of heart image (Fig. 8),

- Figure 34 presents Amplitude,

- Figure 35 presents Phase,

- Figure 36 presents Isotropy,

- Figure 37 presents Theta.

Extracted by SMV of IMF (Fig. 11) obtained by BEMD of bassin image (Fig. 3),

- Figure 38 presents Amplitude,

- Figure 39 presents Phase,

- Figure 40 presents Isotropy,

- Figure 41 presents Theta.

Extracted by SMV of IMF (Fig. 12) obtained by BEMD of abbey texture (Fig. 4),

- Figure 42 presents Amplitude,

- Figure 43 presents Phase,

- Figure 44 presents Isotropy,

- Figure 45 presents Theta.

Extracted by SMV of IMF (Fig. 14) obtained by BEMD of fabric image (Fig. 9),

- Fig. 46 presents Amplitude, 


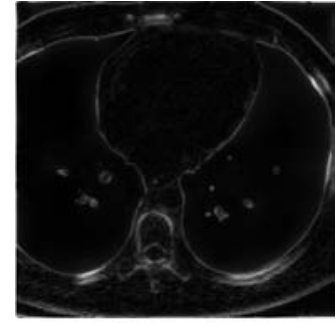

Amplitude of Mode 1

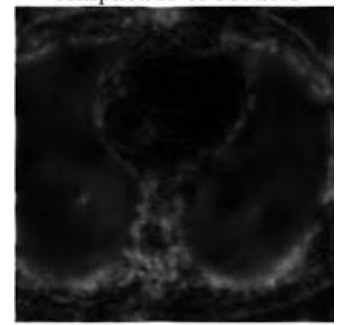

Amplitude of Mode 2

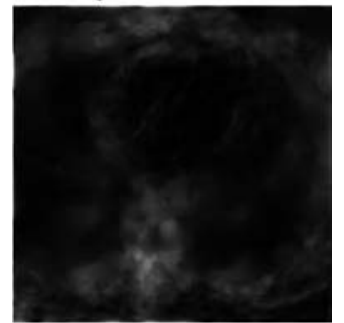

Amplitude of Mode 3

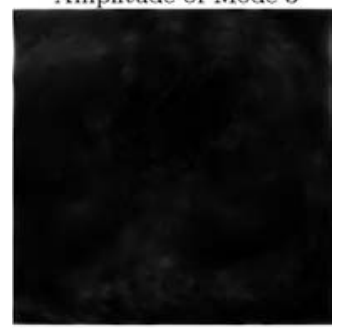

Amplitude of Mode 4

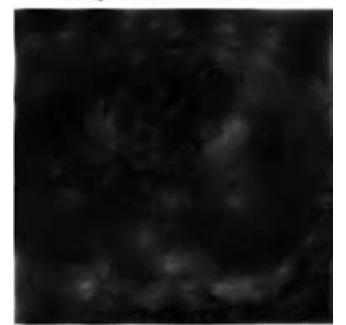

Amplitude of Mode 5

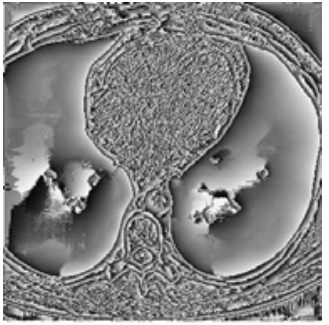

Phase of Mode 1

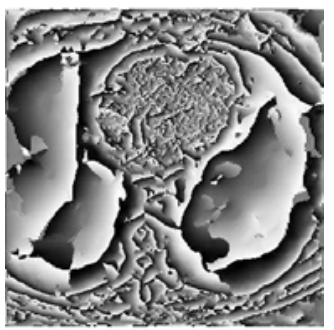

Phase of Mode 2

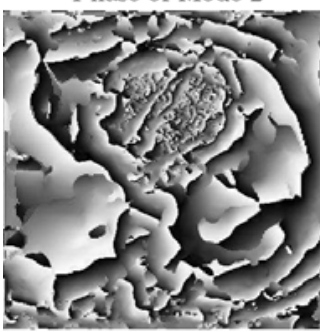

Phase of Mode 3

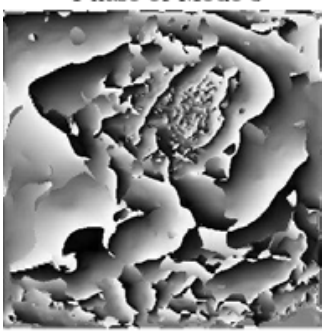

Phase of Mode 4

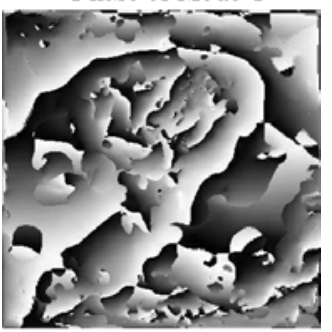

Phase of Mode 5
Fig. 34. Amplitude (extracted from SMV) of IMF (Fig. 20) obtained by BEMD of heart image (Fig. 8).
Fig. 35. Phase (extracted from SMV) of IMF (Fig. 20) obtained by BEMD of heart image (Fig. 8). 


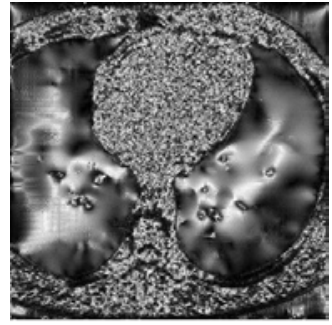

Isotropy of Mode 1

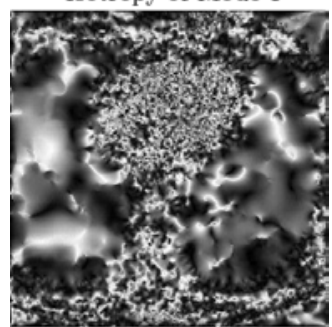

Isotropy of Mode 2

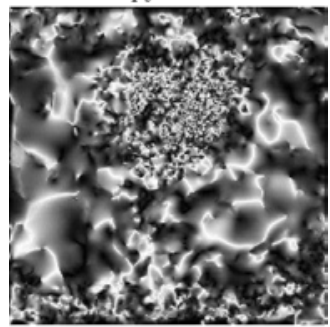

Isotropy of Mode 3

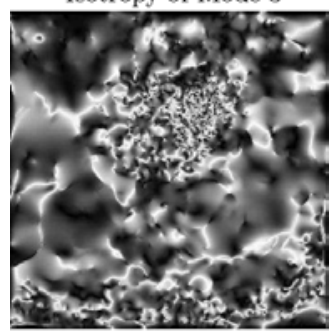

Isotropy of Mode 4

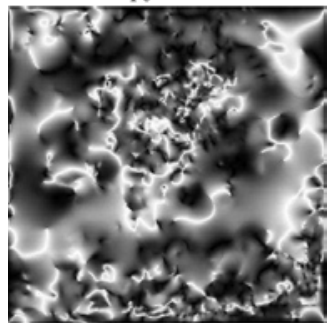

Isotropy of Mode 5

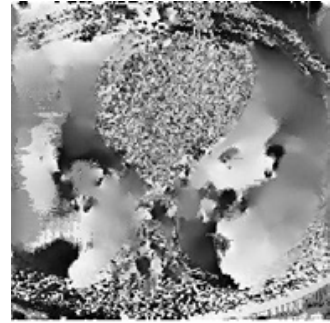

Theta of Mode 1

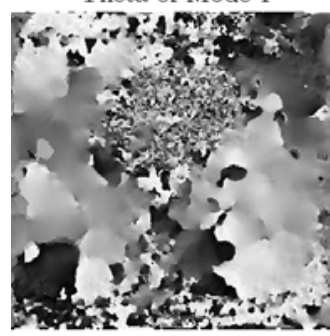

Theta of Mode 2

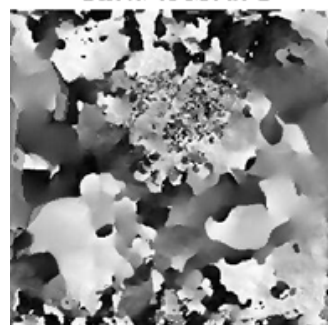

Theta of Mode 3

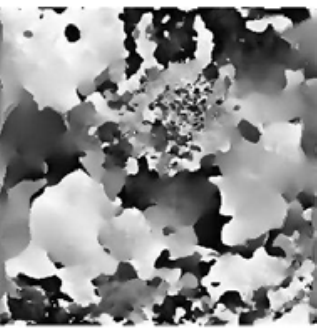

Theta of Mode 4

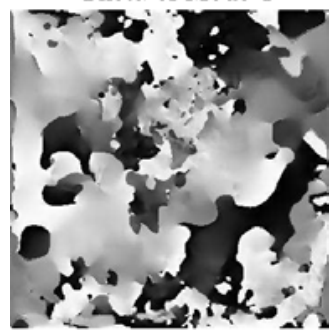

Theta of Mode 5
Fig. 36. Isotropy (extracted from SMV) of IMF (Fig. 20) obtained by BEMD of heart image (Fig. 8).
Fig. 37. Theta (extracted from SMV) of IMF (Fig. 20) obtained by BEMD of heart image (Fig. 8). 


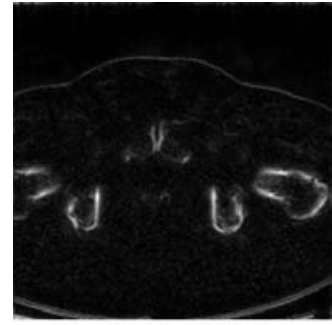

Amplitude of Mode 1

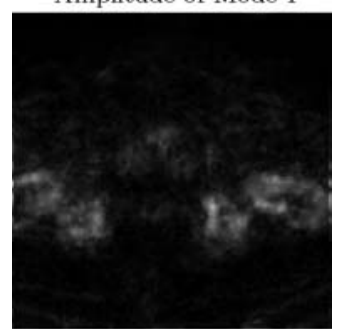

Amplitude of Mode 2

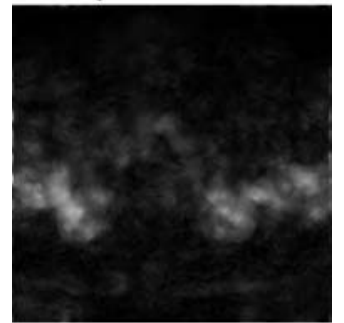

Amplitude of Mode 3

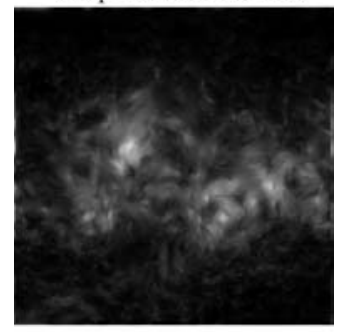

Amplitude of Mode 4

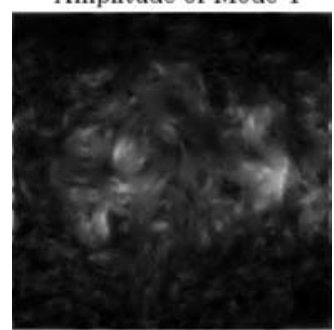

Amplitude of Mode 5

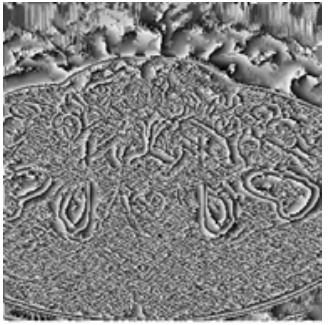

Phase of Mode 1

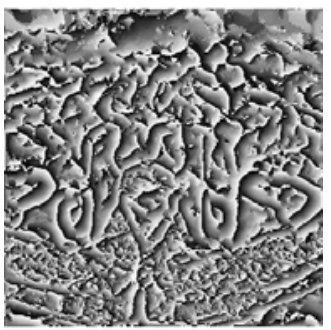

Phase of Mode 2

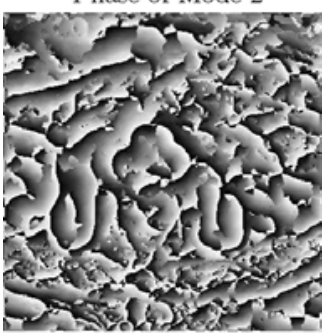

Phase of Mode 3

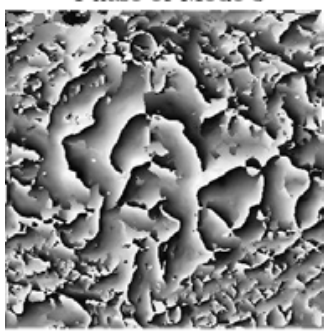

Phase of Mode 4

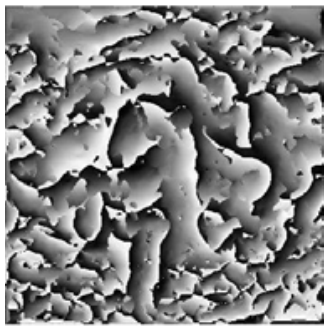

Phase of Mode 5
Fig. 38. Amplitude (extracted from SMV) of IMF (Fig. 11) obtained by BEMD of bassin image (Fig. 3).
Fig. 39. Phase (extracted from SMV) of IMF (Fig. 11) obtained by BEMD of bassin image (Fig. 3). 


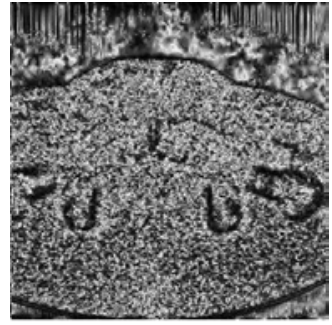

Isotropy of Mode 1

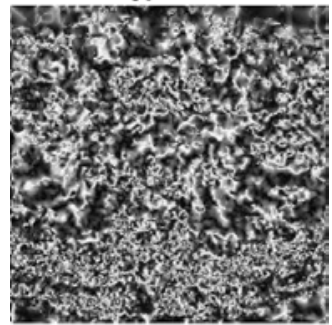

Isotropy of Mode 2

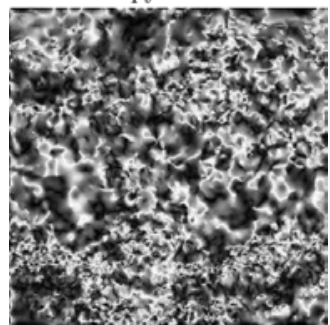

Isotropy of Mode 3

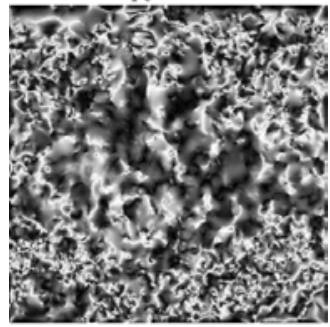

Isotropy of Mode 4

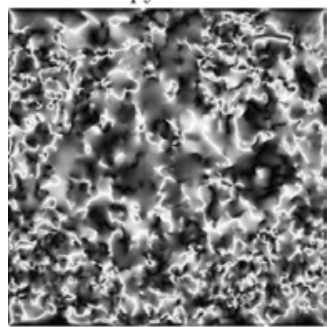

Isotropy of Mode 5

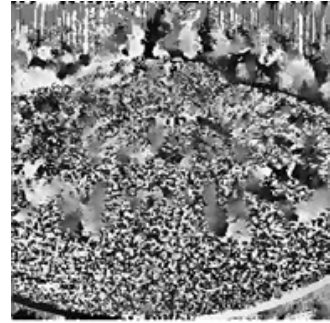

Theta of Mode 1

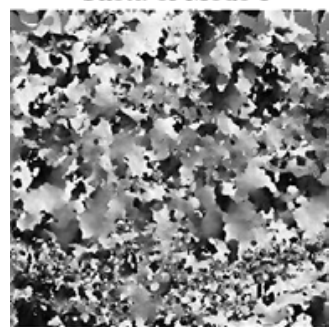

Theta of Mode 2

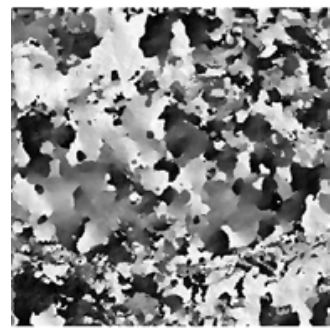

Theta of Mode 3

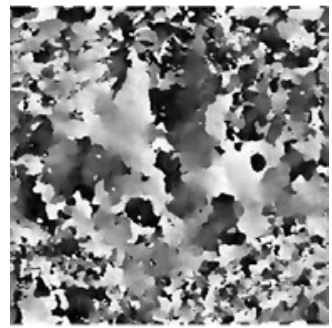

Theta of Mode 4

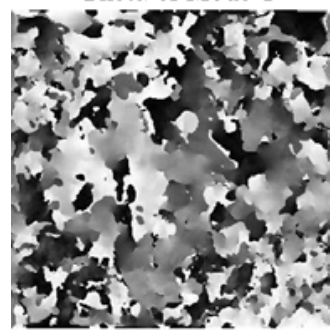

Theta of Mode 5
Fig. 40. Isotropy (extracted from SMV) of IMF (Fig. 11) obtained by BEMD of bassin image (Fig. 3).
Fig. 41. Theta (extracted from SMV) of IMF (Fig. 11) obtained by BEMD of bassin image (Fig. 3). 


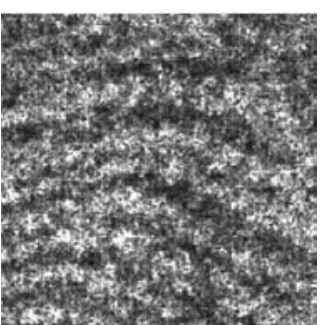

Amplitude of Mode 1

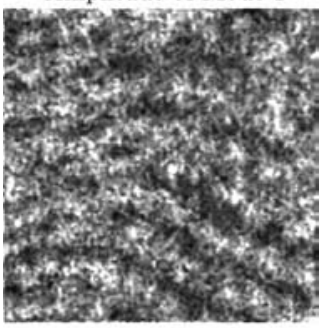

Amplitude of Mode 2

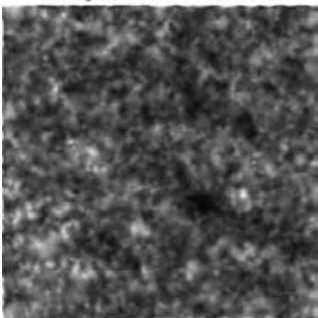

Amplitude of Mode 3

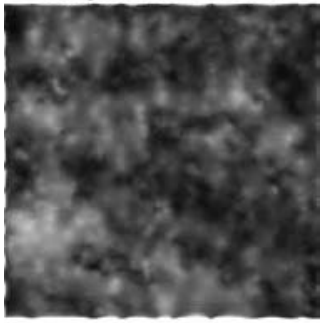

Amplitude of Mode 4

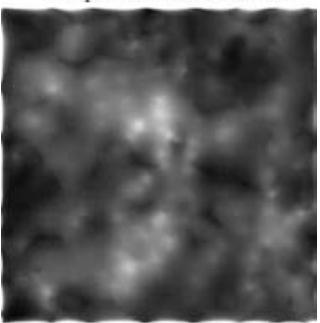

Amplitude of Mode 5

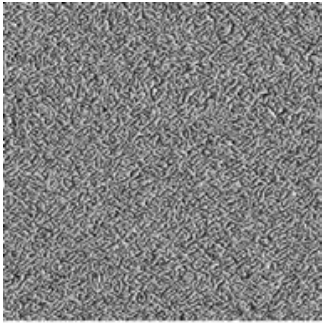

Phase of Mode 1

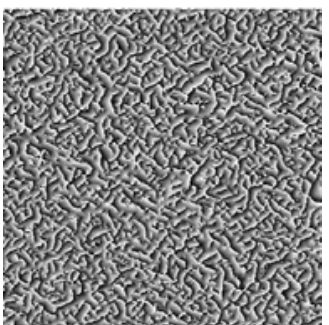

Phase of Mode 2

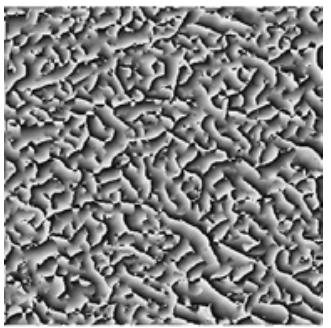

Phase of Mode 3

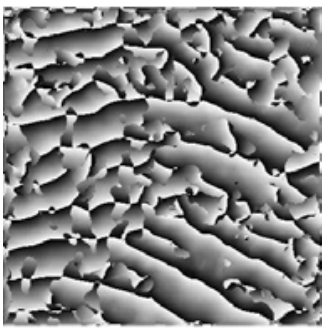

Phase of Mode 4

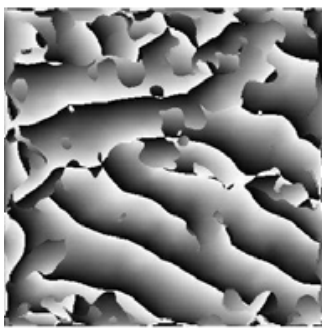

Phase of Mode 5
Fig. 42. Amplitude (extracted from SMV) of IMF (Fig. 12) obtained by BEMD of abbey texture (Fig. 4).
Fig. 43. Phase (extracted from SMV) of IMF (Fig. 12) obtained by BEMD of abbey texture (Fig. 4). 


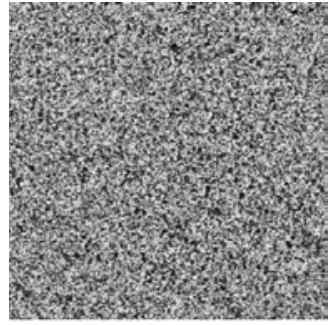

Isotropy of Mode 1

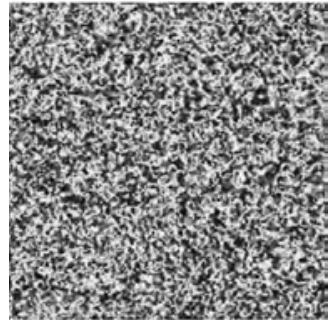

Isotropy of Mode 2

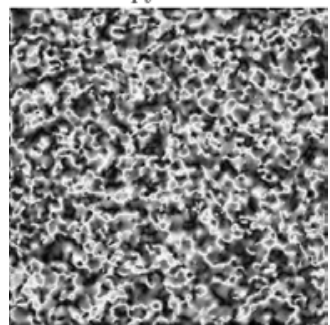

Isotropy of Mode 3

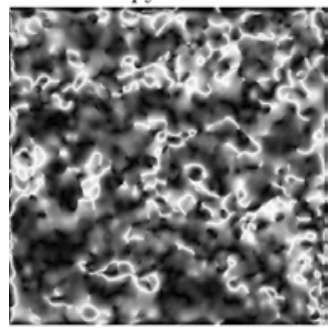

Isotropy of Mode 4

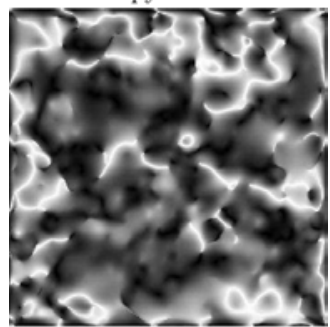

Isotropy of Mode 5

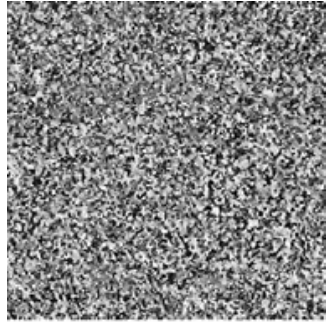

Theta of Mode 1

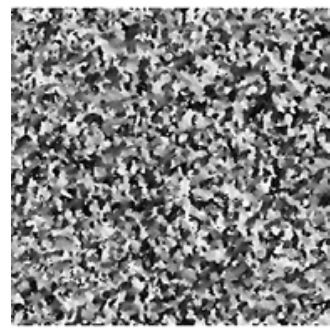

Theta of Mode 2

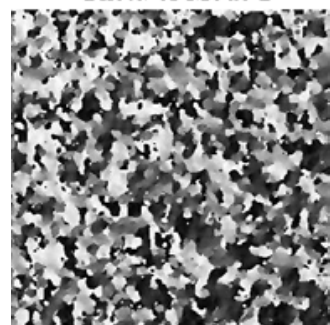

Theta of Mode 3

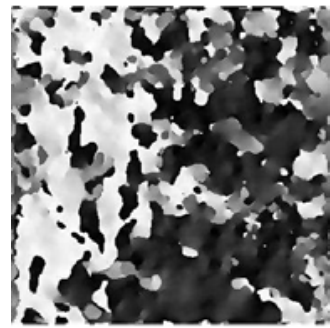

Theta of Mode 4

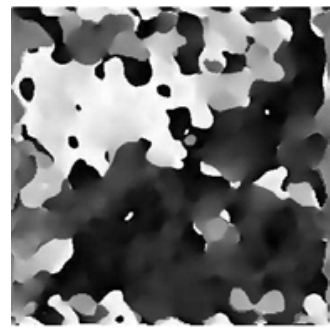

Theta of Mode 5
Fig. 44. Isotropy (extracted from SMV) of IMF (Fig. 12) obtained by BEMD of abbey texture (Fig. 4).
Fig. 45. Theta (extracted from SMV) of IMF (Fig. 12) obtained by BEMD of abbey texture (Fig. 4). 


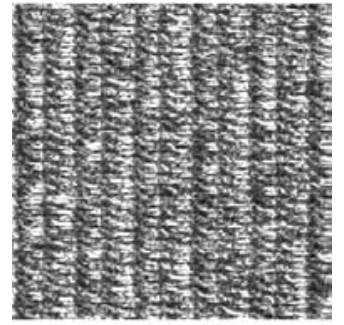

Amplitude of Mode 1

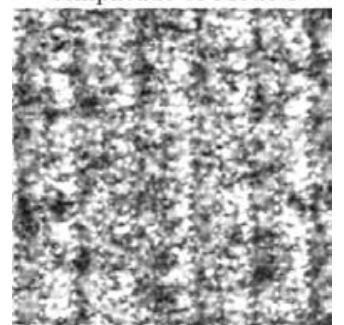

Amplitude of Mode 2

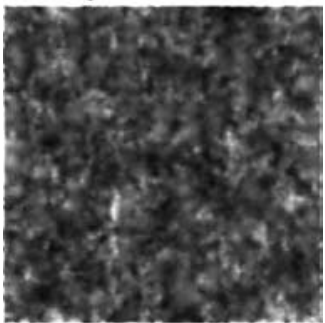

Amplitude of Mode 3

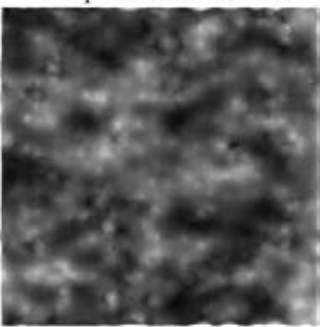

Amplitude of Mode 4

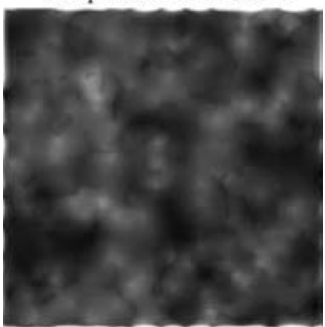

Amplitude of Mode 5

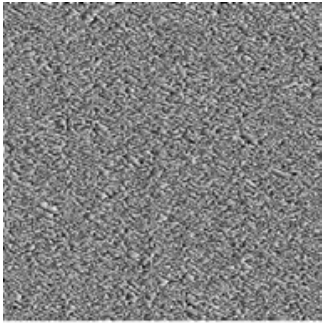

Phase of Mode 1

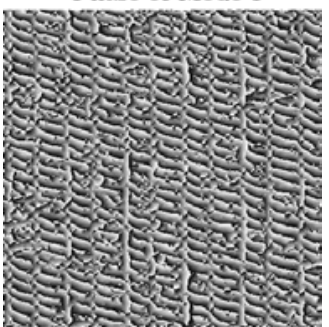

Phase of Mode 2

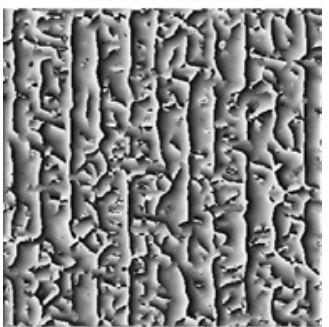

Phase of Mode 3

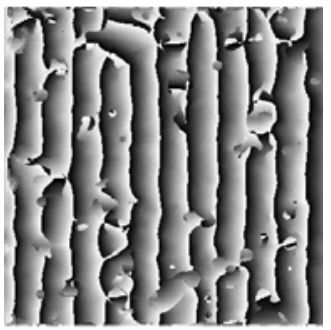

Phase of Mode 4

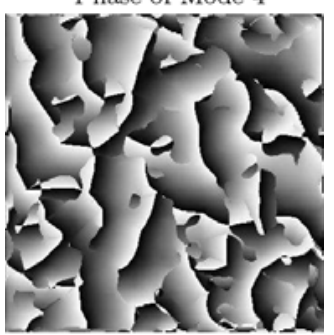

Phase of Mode 5
Fig. 46. Amplitude (extracted from SMV) of IMF (Fig. 14) obtained by BEMD of fabric image (Fig. 9).
Fig. 47. Phase (extracted from SMV) of IMF (Fig. 14) obtained by BEMD of fabric image (Fig. 9). 


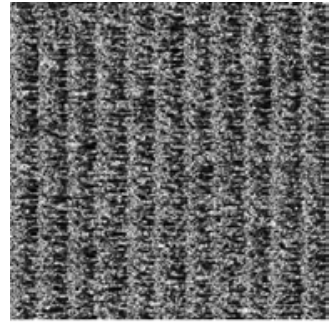

Isotropy of Mode 1

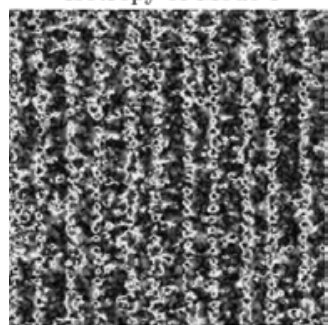

Isotropy of Mode 2

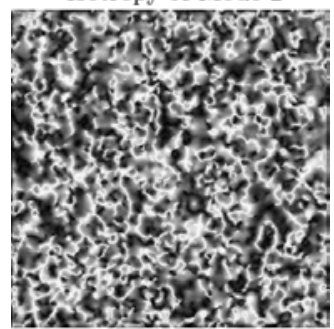

Isotropy of Mode 3

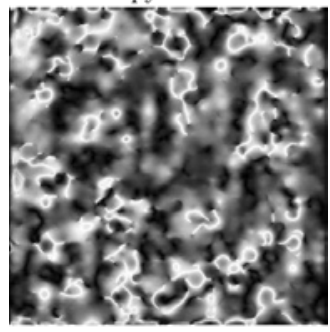

Isotropy of Mode 4

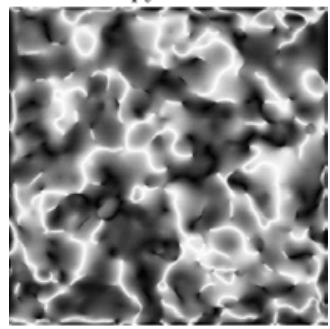

Isotropy of Mode 5

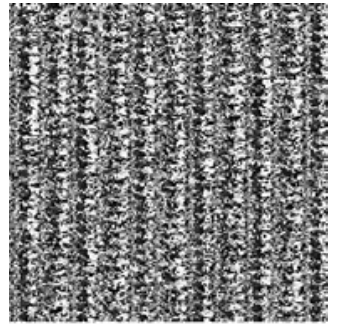

Theta of Mode 1

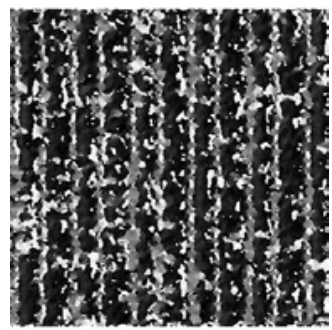

Theta of Mode 2

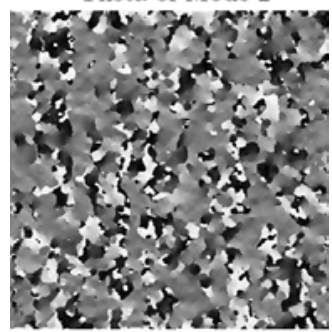

Theta of Mode 3

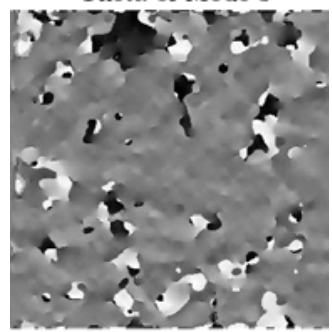

Theta of Mode 4

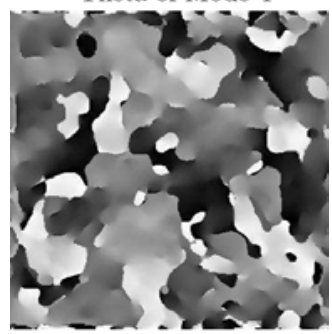

Theta of Mode 5
Fig. 48. Isotropy (extracted from SMV) of IMF (Fig. 14) obtained by BEMD of fabric image (Fig. 9).
Fig. 49. Theta (extracted from SMV) of IMF (Fig. 14) obtained by BEMD of fabric image (Fig. 9). 


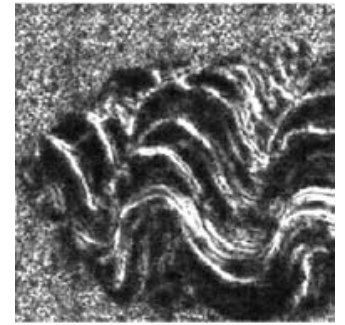

Amplitude of Mode 1

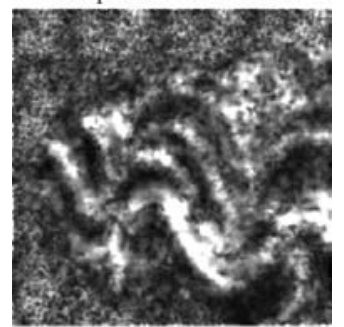

Amplitude of Mode 2

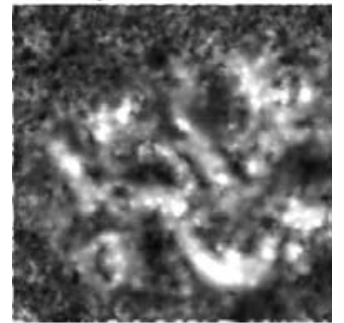

Amplitude of Mode 3

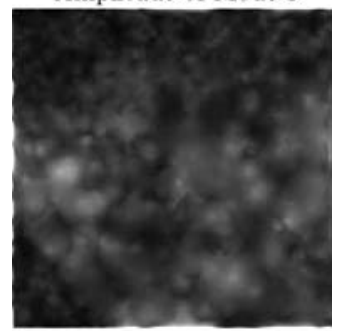

Amplitude of Mode 4

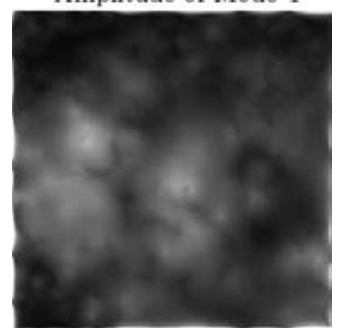

Amplitude of Mode 5

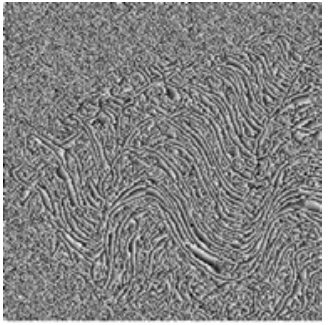

Phase of Mode 1

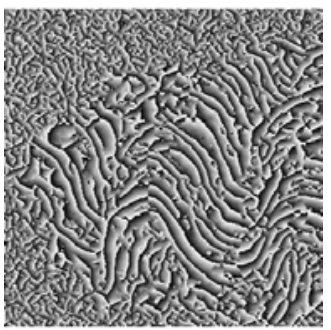

Phase of Mode 2

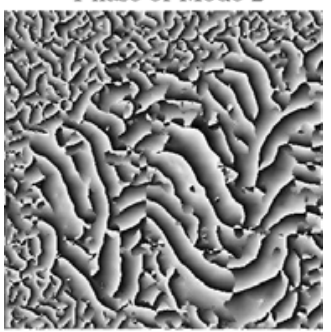

Phase of Mode 3

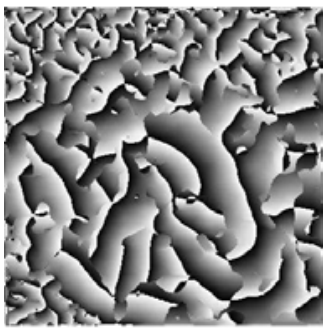

Phase of Mode 4

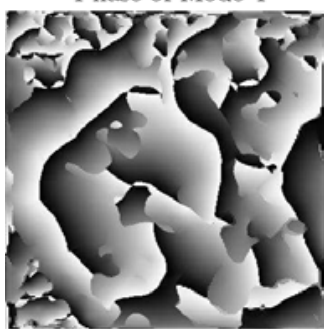

Phase of Mode 5
Fig. 50. Amplitude (extracted from SMV) of IMF (Fig. 15) obtained by BEMD of wavy image (Fig. 10).
Fig. 51. Phase (extracted from SMV) of IMF (Fig. 15) obtained by BEMD of wavy image (Fig. 10). 


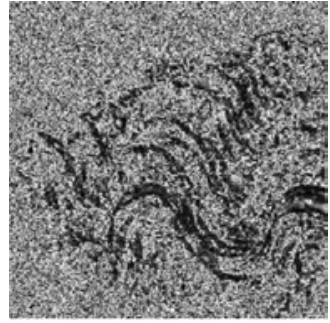

Isotropy of Mode 1

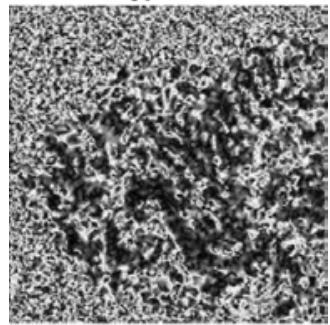

Isotropy of Mode 2

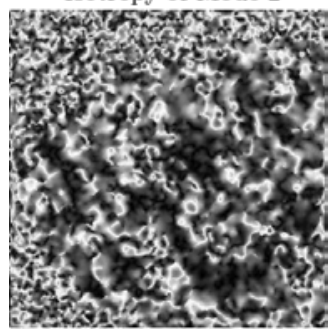

Isotropy of Mode 3

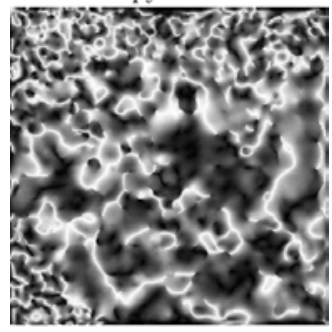

Isotropy of Mode 4

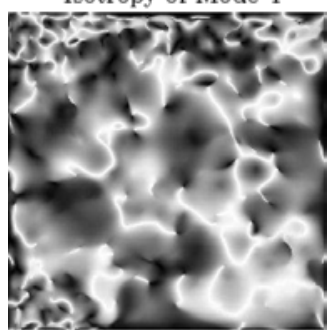

Isotropy of Mode 5

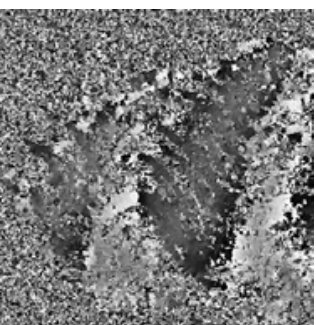

Theta of Mode 1

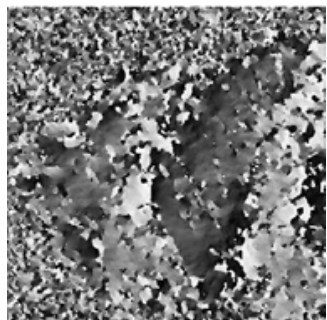

Theta of Mode 2

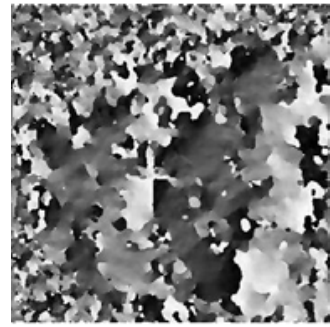

Theta of Mode 3

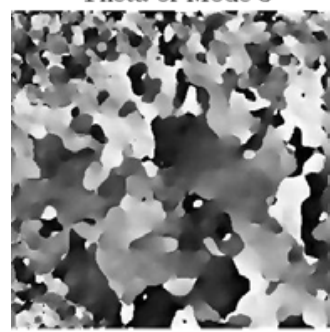

Theta of Mode 4

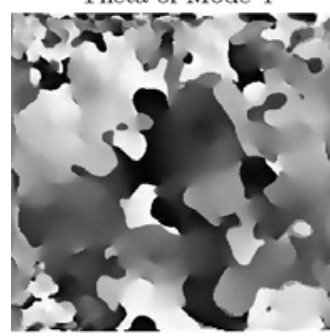

Theta of Mode 5
Fig. 52. Isotropy (extracted from SMV) of IMF (Fig. 15) obtained by BEMD of wavy image (Fig. 10).
Fig. 53. Theta (extracted from SMV) of IMF (Fig. 15) obtained by BEMD of wavy image (Fig. 10). 
- Fig. 47 presents Phase,

- Fig. 48 presents Isotropy,

- Fig. 49 presents Theta.

Extracted by SMV of IMF (Fig. 15) obtained by BEMD of wavy image (Fig. 10),

- Fig. 50 presents Amplitude,

- Fig. 51 presents Phase,

- Fig. 52 presents Isotropy,

- Fig. 53 presents Theta.

Although all the local features of all the 2D-IMF are not really useful in a later stage of segmentation, some can-to be relevant. Indeed, of the uniform regions are observable and can correspond to the required structures.

\section{Analytical Approach}

This work have been published in Ref. 22. The mean envelope originally proposed by Huang is obtained by the difference between the upper and lower envelopes which connect, respectively, all the maxima or all minima by cubic spline. This iteration procedure (called the sifting process) is repeated until strictly zero mean.

This analytical approach which replace the sifting process is based on a parabolic PDE. In order to implement sifting procedure in a PDE-based framework, the following processes are based on the definition of characteristics fix points of a function: (i) turning points, (ii) curvature points. The present research focuses its interest on turning points, which are minima, maxima, and inflexion points, defined by the values of their first and/or second derivatives. The discussions in the following two sub-sections make use of fourth-order parabolic equations of the form:

\subsection{PDE-based formulation in $1 D$}

A possible form for fourth-order diffusion equation is

$$
\frac{\partial s(x, t)}{\partial t}=-\frac{\partial}{\partial x}\left(g(x, t) \frac{\partial^{3} s(x, t)}{\partial x^{3}}\right),
$$

where $g(x, t)$ is the diffusivity function possibly depending on both position and time, and where the time variable is artificial, and measures the degree of processing (e.g., smoothing) of the signal, as opposed to a real time. Equation (21) can be viewed as a Long-range diffusion (LRD) equation (see for example Ref. 72, p. 244), with thresholding function $g(x)$ depending only on position (constant in time) and more precisely on some characteristic fix points of the signal to decompose. After derivation equation (21) read:

$$
s_{t}(x, t)=-\partial_{x}^{1} g(x) \partial_{x}^{3} s(x, t),
$$


where the subscript $t$ denotes partial differentiation with respect to the variable $t$ and $\partial_{x}^{q}$ denotes partial differentiation of order $q$ with respect to the variable $x$. In the following we use the notation $s_{0}(x)=s(x, t=0)$ for initial condition and $s_{\infty}(x)=s(x, t=\infty)$ for asymptotic solution of Eq. (21).

In order to implement sifting procedure in a PDE-based framework, the following processes are based on the definition of characteristic fix points of a function: (i) turning-points; (ii) curvature-points. Here, we are interesting in turning points that are minima, maxima, and inflexion points, defining by the values of their first and/or second derivatives. We have to define a coupled PDEs system in place of sifting process to estimate lower and upper envelopes.

\subsubsection{A coupled PDEs system}

A simple method to estimate mean-envelope is to formulate a coupled PDEs system to mimic Huang's sifting process based on upper and lower envelopes estimation. Turning points are here respectively maxima and minima of the signal to be decomposed. This coupled PDEs system, based on Eq. (21) reads (see also Ref. ${ }^{22}$ for a less general PDE formulation):

$$
\left\{\begin{array}{l}
s_{t}^{+}(x, t)=-\delta_{x}^{1}\left[g^{+}\left(\delta_{x}^{1} s_{0}(x), \delta_{x}^{2} s_{0}(x)\right) \delta_{x}^{3} s^{+}(x, t)\right] \\
s_{t}^{-}(x, t)=-\delta_{x}^{1}\left[g^{-}\left(\delta_{x}^{1} s_{0}(x), \delta_{x}^{2} s_{0}(x)\right) \delta_{x}^{3} s^{-}(x, t)\right]
\end{array}\right.
$$

After convergence of system (23) asymptotic solutions $s_{\infty}^{+}(x)$ and $s_{\infty}^{-}(x)$, stand respectively for upper and lower envelops of signal $s_{0}$. Hence, mean-envelope of $s_{0}$ is obtained by:

$$
s_{\infty}(x)=\frac{1}{2}\left[s_{\infty}^{+}(x)+s_{\infty}^{-}(x)\right]
$$

In Eq. (23), stopping functions, $g^{ \pm}$, depend on both first- and second-order signal derivatives, with $0 \leq g^{ \pm} \leq 1$. For example, a good choice for stopping functions seems to be

$$
g^{ \pm}(x)=\frac{1}{9}\left[\left|\operatorname{sgn}\left(\delta_{x}^{1} s_{0}(x)\right)\right| \pm \operatorname{sgn}\left(\delta_{x}^{2} s_{0}(x)\right)+1\right]^{2} .
$$

In such a way, $g^{+}=0$ and $\delta_{x}^{1} g^{+}=0$ at maxima of $s_{0}$, in the same way $g^{-}=$ 0 and $\delta_{x}^{1} g^{-}=0$ at minima of $s_{0}$. So, LRD acts only between two consecutive maxima (resp. minima) points until fourth-order derivative of $s(x, t)$ is canceled. Since, stopping functions are piecewise constant, after convergence the resulting signal $s_{\infty}^{+}(x)$ (resp. $s_{\infty}^{-}(x)$ ) is a piecewise cubic polynomial curve interpolating the successive maxima (resp. minima) of signal. In Eq. (24) sign function, $\operatorname{sgn}(z)$, is replaced by a regularized version. A possible expression is given by $\operatorname{sgn}_{\alpha}(z)=$ $2 / \pi \arctan (\pi z / \alpha)$. 


\subsubsection{Interpolation with tension}

A more general form for Eq. (22) is

$$
s_{t}(x, t)=\delta_{x}^{1}\left[g(x)\left(\alpha \delta_{x}^{1} s(x, t)-(1-\alpha) \delta_{x}^{3} s(x, t)\right)\right] .
$$

So, in this form, $\alpha$ is the tension parameter, and ranges from 0 to 1 . Zero tension, $\alpha=0$, leads to the biharmonic equation form (22) and corresponds to the minimum curvature construction for upper and lower envelopes. The case $\alpha=1$ corresponds to infinite tension (piecewise linear envelopes).

\subsection{Numerical resolution}

Numerical resolution for coupled PDEs system based on Eq. (25) is implemented with a Crank-Nicolson scheme (semi or fully implicit) or Du Fort and Frankel scheme. Noting that a particular attention is made for derivatives of $s_{0}$ in the defintion of $g(x)$ :

$$
g(x)=g\left(D_{1} s_{0}(x), D_{2} s_{0}(x)\right)
$$

where $g=g^{ \pm}$, and $D_{1} z=\operatorname{minmod}\left(D^{+} z, D^{-} z\right), D_{2} z=D^{+} D^{-}$, where $D^{+}$and $D^{-}$ are forward and backward first difference operators on the $x$-dimension, and where $\operatorname{minmod}(a, b)$ stands for the minmod limiter $\operatorname{minmod}(a, b)=\frac{1}{2}[\operatorname{sgn}(a)+\operatorname{sgn}(b)]$. $\min (|a|,|b|)$.

\subsection{Results}

To give a concise presentation, the following illustrating results (published in Ref. 22) were obtained using coupled PDEs process. Equation is also capable of producing similar results but requires a careful, and hence time consuming, attention on step-time value $\delta t$. Contrary to the solutions of Eq. (11) of Ref. 22, solutions are less sensitive to step time. All IMFs were obtained in a small number (lower than 8) of iterations in the PDE-based sifting procedure and with a constant time step (for example $\delta t=20$ ) to assure relatively fast convergence of the process. In order to make a proper comparison the examples described in Ref. 36 are again considered.

Figure 54 illustrates the modewise decomposition, of a Dirac impulse. It can be easily seen that the wavelet-like form of the successively extracted five first components are in agreement with the results reported in Ref. 36. The first example of signal decomposition consists of a sum of two triangular waveforms and a tone (presented in Fig. 55). The second example is a composite signal originating from the superposition of two sinusoidal FM signals and one Gaussian logon (presented in Fig. 56). In this case, the components overlap in both time and frequency, thus 


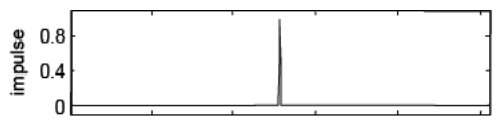

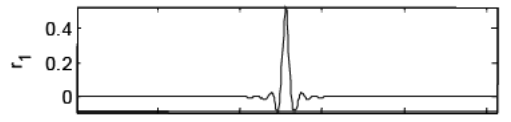
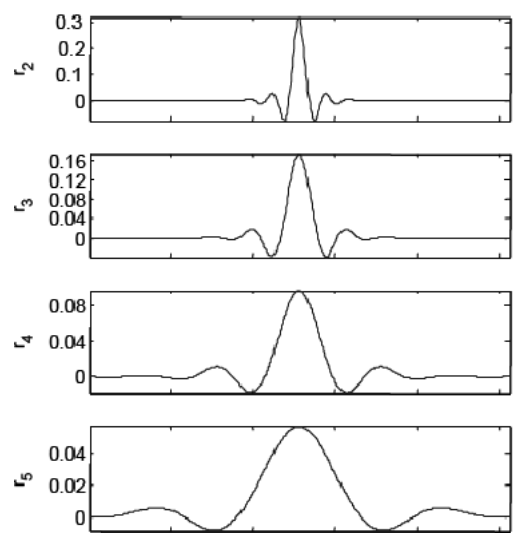
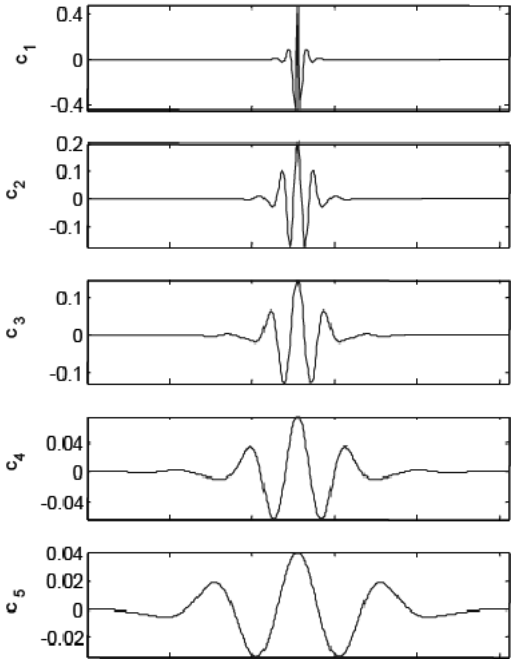

Fig. 54. Decomposition of a Dirac impulse. Five first components (right column) and residuals (left column) of the decomposition of an impulse signal (shown in the top plot). The components labeled c1 to c5 (right) are equivalent to impulse responses of a filter bank (compare with the results from EMD reported in Ref. 37). First published in Ref. 22.
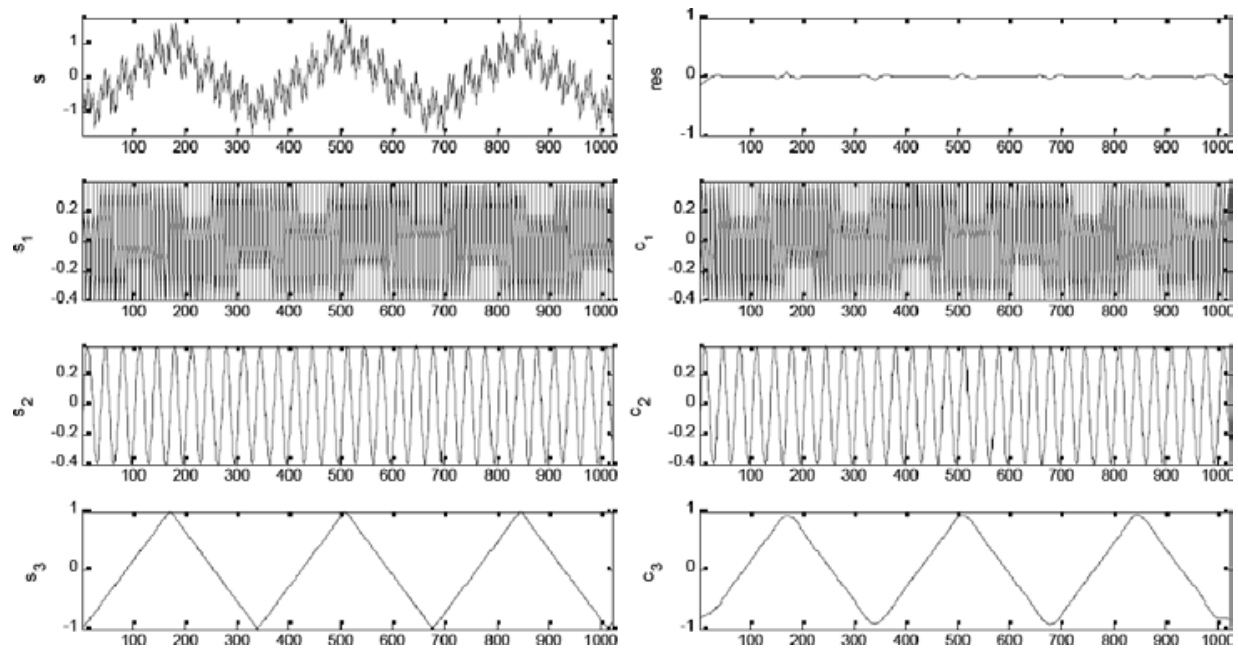

Fig. 55. PDE-based signal decomposition. In this first example, a composite signal (left, first row), resulting from the superposition of two triangular (s1 and s3) waveforms and one tone (s2), is decomposed into its three expected elementary components labeled c1 to c3 (right, rows 2-4). Residual component of the decomposition is also given in the first row (compare these results with those obtained using EMD algorithm, Fig. 2 in Ref. 83). First published in Ref. 22. 

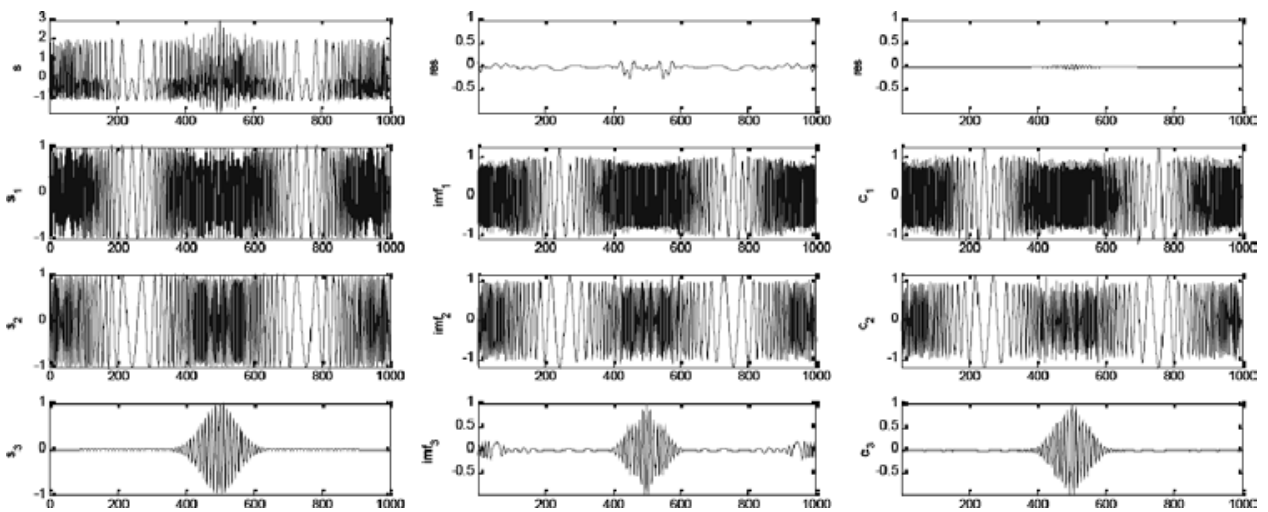

Fig. 56. PDE-based signal decomposition. In this second example, a composite signal (left column, first row), resulting from the superposition of two sinusoidal FM signals (s1 and s2) and one Gaussian logon (s3), is decomposed in its three expected elementary components labeled c1 to c3 (right column, rows 2-4). The residual component of the decomposition is also given in the first row. For comparison, IMF and the residual resulting from classical EMD is also shown (IMFs are labeled as imf1 to imf3, shown in the middle column). First published in Ref. 22.

disabling the components to be separated by any non-adaptive filtering technique. In all these examples, both linear and nonlinear oscillations are effectively separated.

\section{Conclusion}

In this paper, we address the topics of multiscale representation obtained by BEMD, and image analysis.

We extend the EMD to 2D-data (i.e., images), the so-called bidimensional EMD (BEMD), the process being called 2D-sifting process.

We propose an algorithm to generate fractional Gaussian noise (fGn), and fractional Brownian motion (fBm) of Hurst exponent $H$ from IMFs obtained from EMD of a White noise, i.e., ordinary Gaussian noise (fGn with $H=1 / 2$ ).

We analyze each 2D-IMF obtained by BEMD by studying local properties (amplitude, phase, isotropy, and orientation) extracted from the monogenic signal of each one of them. The monogenic signal proposed by Felsberg et al. is a 2D-generalization of the analytic signal, where the Riesz transform replaces the Hilbert transform. The structure multivector (SMV) is an operator for analyzing the local structure of an image. It combines ideas from the structure tensor, steerable filters, and quadrature filters where the advantages of all three approaches are brought into a single method by means of geometric algebra.

Deléchelle et al. proposed an analytical approach (formulated as a partial differential equation) for sifting process. This PDE-based approach is applied on signals. The analytical approach has a behavior similar to that of the EMD proposed by Huang. 


\section{References}

1. P. Abry et al., Wavelets for the analysis estimation and synthesis of scaling data, in Self-Similar Network Traffic and Performance Evaluation, eds. K. Park and W. Willinger (Wiley, New York, 2000).

2. I. Amidror, Scattered data interpolation methods for electronic imaging systems: A survey, J. Electronic Imag. 11(2) (2002) 157-176.

3. F. Andreu, C. Ballester, V. Caselles and J. M. Mazon, Minimizing total variation flow, Different. Integral Eq. 14 (2001) 321-360.

4. G. Aubert and P. Kornprobst, Mathematical Problems in Image Processing: Partial Differential Equations and the Calculus of Variations, in Applied Mathematical Sciences, Vol. 147 (Springer, New York, 2002).

5. J. M. Bardet et al., Generators of long-range dependent processes: A survey, longrange dependence, in Theory and Applications (Birkhauser, Basel, 2002).

6. R. Barnhill, Representation and approximation of surfaces, in Mathematical Software III, ed. J. R. Rice (Academic Press, New York, 1997), pp. 68-119.

7. R. H. Barthels, J. C. Beatty and B. A. Barsky, An Introduction to Splines for Use in Computer Graphics and Geometric Modeling (Morgan Kaufmann Publishers, 1987).

8. R. K. Beatson and W. A. Light, Fast evaluation of radial basis functions: Methods for 2-D polyharmonic splines, Tech. Rep. 119, Mathematics Department, Univ. of Canterbury, Christchurch, New Zealand, Dec. 1994.

9. A. Benassi and S. Deguy, Multi-scale fractional Brownian motion: Definition and identification, LLAIC Technical Report (1999), p. 83.

10. M. Bertram, X. Tricoche and H. Hagen, Adaptive smooth scattered-data approximation for large-scale terrain visualization, in Proceedings of the Symposium on Data Visualisation 2003 (Eurographics Association, 2003), pp. 177-184.

11. M. Bertram, M. A. Duchaineau, B. Hamann and K. I. Joy, Generalized b-spline subdivision-surface wavelets for geometry compression, IEEE Trans. Visual. Comput. Graph. 10(3) (2004) 326-338.

12. S. Beucher and F. Meyer, The morphological approach to segmentation: The watershed transformation, in Mathematical Morphology in Image Processing, ed. Edward R. Dougherty (Marcel Dekker, Inc., New York, 1993), Chap. 12, pp. 433-481.

13. S. Beucher, Geodesic reconstruction, saddle zones and hierarchical segmentation, Image Anal. Stereol. 20 (2001) 137-141.

14. M. D. Buhmann, Radial basis functions, Acta Numerica. (2000) 1-38.

15. T. Bülow, Hypercomplex spectral signal representations for the processing and analysis of images, Thesis, Christian-Albrechts-Universität zu Kiel, Institut fr Informatik und Praktische Mathematik, 1999.

16. T. Bülow and G. Sommer, Hypercomplex signals: A novel extension of the analytic signal to the multidimensional case, IEEE Trans. Signal Process. 49(11) (2001) 2844-2852.

17. J. C. Carr, W. R. Fright and R. K. Beatson, Surface interpolation with radial basis functions for medical imaging, Computer Graphics Proceedings, Annual Conference Series (SIGGRAPH 2001) (2001), pp. 67-76.

18. A. Certain, J. Popovic, T. DeRose, T. Duchamp, D. Salesin and W. Stuetzle, Interactive multiresolution surface viewing, Computer Graphics (Proceedings of SIGGRAPH '96) 30 (1996) 97-115.

19. B. B. Chaudhuri, N. Sarkar and P. Kundu, Improved fractal geometry based on texture segmentation technique, IEEE Proceedings-E 140 (1993) 233-241.

20. M. Daehlen and V. Skyth, Modelling non-rectangular surfaces using box-splines, D. C. Handscomb, Mathematics of Surfaces III (1989), pp. 287-300. 
21. C. Damerval, S. Meignen and V. Perrier, A fast algorithm for bidimensional, IEEE Signal Process. Lett. 12(10) (2005).

22. E. Delechelle, J. Lemoine and O. Niang, Empirical mode decomposition: An analytical approach for sifting process, IEEE Signal Process. Lett. 12 (2005) 764-767.

23. É. Deléchelle, J. C. Nunes and J. Lemoine, Empirical mode decomposition synthesis of fractional processes in 1D- and 2D-space, Image Vis. Comput. 23(9) (2005) 799806.

24. M. Deriche and A. H. Tewfik, Maximum likelihood estimation of the parameters of discrete fractionally differenced Gaussian noise processes, IEEE Trans. Signal Process. 41 (1993) 2977-2989.

25. S. Didas, J. Weickert and S. Burgeth, Stability and local feature enhancement of higher order nonlinear diffusion filtering, Pattern Recognition (DAGM 2005), pp. 451-458.

26. J. Duchon, Splines minimizing rotation-invariant semi-norms in Sobolevspaces, in Constructive Theory of Functions of Several Variables, eds. W. Schempp and K. Zeller (Springer, Berlin, 1977), pp. 85-100.

27. R. Duits, L. M. J. Florack, J. de Graaf and B. M. ter Haar Romeny, On the axioms of scale-space theory, J. Imag. Vis. 20(3) (2004) 267-298.

28. D. Dunn, W. E. Higgins and J. Wakeley, Texture segmentation using 2-D Gabor elementary functions, IEEE Trans. Pattern. Anal. Mach. Intell. 16(2) (1994) 130-149.

29. P. Embrechts and M. Maejima, Selfsimilar Processes (Princeton University Press, 2002).

30. K. J. Falconer, The Geometry of Fractal Sets (Cambridge University Press, New York, 1985).

31. M. Felsberg and G. Sommer, The monogenic signal, IEEE Trans. Signal Process. 49 (2001).

32. M. Felsberg and G. Sommer, Structure multivector for local analysis of images, in Proc. Dagstuhl Workshop on Theoretical Foundations of Computer Vision, eds. R. Klette, T. Huang and G. Gimelfarb, Multi-Image Analysis, Vol. 2032 of LNCS (Springer-Verlag, Berlin, 2001), pp. 93-104.

33. M. Felsberg and G. Sommer, The monogenic scale-space: A unifying approach to phase-based image processing in scale-space, J. Math. Imag. Vis. 21 (2004) 5-26.

34. M. Felsberg, Low-Level image processing with the structure multivector, $\mathrm{PhD}$ thesis, Institute of Computer Science and Applied Mathematics ChristianAlbrechts-University of Kiel, TR No. 0203, Available at http://www.informatik.unikiel.de/reports/2002/0203.html.

35. A. Finkelstein and D. H. Salesin, Multiresolution curves, Computer Graphics (Proceedings of SIGGRAPH 94) 28 (1994) 261-268.

36. P. Flandrin, Some aspects of Huang's empirical mode decomposition, from interpretation to applications, Int. Conf. Computat. Harmonic Anal. CHA-04 (invited talk), Nashville, TN (2004).

37. P. Flandrin and P. Gonçalvès, Sur la décomposition modale empirique, GRETSI, Paris, 2003.

38. P. Flandrin, Wavelets analysis and synthesis of fractional Brownian motions, IEEE Trans. Inform. Theory 38 (1992) 910-917.

39. P. Flandrin and P. Gonçalvès, Empirical mode decompositions as a data-driven wavelet-like expansions, Int. J. Wavelets, Multires. and Info. Proc., in press. 
40. P. Flandrin, P. Gonçalvès and G. Rilling, Empirical mode decomposition as a filter bank, IEEE Sig. Proc. Lett. 11(2) (2004) 112-114.

41. D. R. Forsey and R. H. Bartels, Surface fitting with hierarchical splines, ACM Trans. Graphics 14(2) (1995) 134-161.

42. R. Franke and G. M. Nielson, Scattered data interpolation and applications: A tutorial and survey, in Geometric Modelling: Methods and Their Application, eds. H. Hagen and D. Roller (Springer-Verlag, Berlin, 1991), pp. 131-160.

43. N. Gache, P. Flandrin and D. Garreau, Fractal dimension estimators for fractal Brownian motion, IEEE ICASSP 91 (1991) 3557-3560.

44. A. Glassner, Principles of Digital Image Synthesis (Morgan Kaufmann, San Francisco, California, 1995).

45. G. H. Granlund and H. Knutsson, Signal Processing for Computer Vision (Kluwer Academic Publishers, Dordrecht, 1995).

46. S. L. Hahn, Hilbert Transforms in Signal Processing (Artech. House, Boston, London, 1996).

47. M. Hansen, Stereosehen - ein verhaltensbasierter Ansatz, PhD thesis, Inst. f. Inf. u. Prakt. Math. der Christian-Albrechts-Universitat Kiel (1998).

48. R. L. Hardy, Multiquadratic equations of topography and other irregular surfaces, J. Geophys. Res. 76(8) (1971) 1905-1915.

49. H. Hoppe, T. DeRose, T. Duchamp, M. Halstead, M. Jin, J. McDonald, J. Schweitzer and W. Stuetzle, Piecewise smooth surface reconstruction, Computer Graphics (SIGGRAPH '94 Conf. Proc.), (1994), pp. 295-302.

50. J. Hoschek and D. Lasser, Computer Aided Geometric Design (A.K. Peters, Ltd., Wellesley, Mass, 1993).

51. N. E. Huang, Z. Shen, S. R. Long, M. C. Wu, H. H. Shih, Q. Zheng, N.-C. Yen, C. C. Tung and H. H. Liu, The empirical mode decomposition and the Hilbert spectrum for non-linear and non-stationary time series analysis, Proc. Roy. Soc. Lond. A 454 (1998) 903-995.

52. N. E. Huang, M. L. Wu, W. Qu, S. R. Long and S. S. P. Shen, Applications of HilbertHuang transform to non-stationary financial time series analysis, Appl. Stochastic Models Business Industry 19 (2003) 245-268.

53. B. Jähne, Digitale Bildverarbeitung (Springer-Verlag, Berlin, 1997).

54. R. L. Kashyap and P. M. Lapsa, Synthesis and estimation of random fields using long-correlation models, IEEE Trans. Pattern Anal. Mach. Intell. 6 (1984) 800-809.

55. B. Klinkenberg and M. F. Goodchild, The fractal properties of topography: A comparison of methods, Earth Surface Processes and Landforms 17 (1992) 217-234.

56. N. Kojekine, V. Savchenko, D. Berzin and I. Hagiwara, Software Tools for Compactly Supported Radial Basis Functions, in CGIM 2001, IASTED Fourth International.

57. P. Kovesi, Image features from phase congruency, Videre: A J. Comput. Vis. Res. 1(3) (1999).

58. P. Kovesi, Invariant measures of image features from phase information, $\mathrm{PhD}$ thesis, University of Western Australia (1996).

59. S. Lee, G. Wolberg and S. S. Shin, Scattered data interpolation with multilevel b-plines, IEEE Trans. Vis. Comp. Graph. 3(3) (1997).

60. W. Light, Using radial functions on compact domains, in Wavelets, Images and Surface Fitting, eds. P. J. Laurent et al. (AL Peters Ltd., 1994), pp. 351-370.

61. A. Linderhed, 2D empirical mode decompositions in the spirit of image compression, in Wavelet and Independent Component Analysis Applications IX, SPIE Proceedings, Vol. 4738 (2002), pp. 1-8. 
62. A. Linderhed, Image compression based on empirical mode decomposition, Uppsala, March 11-12, Proc. of SSAB 04 Symp. on Image Analysis (2004), pp. 110-113.

63. A. Linderhed, Variable sampling of the empirical mode decomposition of twodimensional signals, Int. J. Wavelets, Multiresolution Inform. Processing, preprint.

64. Z. Liu and S. Peng, Estimation of image fractal dimension based on empirical mode decomposition, in Advanced Concepts for Intelligent Vision Systems (ACIVS), Aug. 31-Sept., Brussels, Belgium.

65. Z. Liu and S. Peng, Texture segmentation using directional Empirical Mode Decomposition, in IEEE International Conference on Image Processing (ICIP), Singapore, 24-27 October 2004.

66. S. K. Lodha and R. Franke, Scattered data techniques for surfaces, in Proc. Dagstuhl Conf. Scientific Vizualization, eds. H. Hagen, G. M. Nielson and F. Post (1999), pp. $182-222$.

67. T. Lundahl et al., Fractional Brownian motion: A maximum likelihood estimator and its applications to image texture, IEEE Trans. Med. Imag. 5 (1986) 152-161.

68. B. B. Mandelbrot, The Fractal Geometry of Nature (Freeman, San Francisco, 1982).

69. B. B. Mandelbrot and N. Van Ness, The fractional Brownian motions, fractional noises and applications, SIAM Rev. 10 (1968) 422-436.

70. D. Marr and E. C. Hildreth, Theory of edge detection, Proc. Roy. Soc. Lond. B 207 (1980) 187-217.

71. B. Morse, T. S. Yoo, P. Rheingans, D. T. Chen and K. R. Subramanian, Interpolating implicit surfaces from scattered surface data using compactly supported radial basis functions, in Proc. Shape Modeling Conf., Genova, Italy, (2001), pp. 89-98.

72. J. D. Murray, Mathematical Biology, Vol. 19 (Springer-Verlag, New York, 1993).

73. G. M. Nielson, Scattered data modeling, IEEE Comput. Graph. Appl. (1993) 60-70.

74. J. C. Nunes, Analyse multiéchelle d'images. Application à l'angiographie rétinienne et à la DMLA, Thesis, Université Paris 12, France.

75. J. C. Nunes, Y. Bouaoune, E. Deléchelle, O. Niang and P. Bunel, Image analysis by bidimensional empirical mode decomposition, Image and Vision Computing 21, (2003) 1019-1026.

76. J. C. Nunes, S. Guyot and E. Deléchelle, Texture analysis based on local analysis of the bidimensional empirical mode decomposition, J. Mach. Vision Appl. (MVA) 16(3) (2005) 177-188.

77. P. J. Oonincx, Empirical mode decomposition: A new tool for S-wave detection, CWI Reports of Probability, Networks and Algorithms (PNA), PNA-R0203.

78. W. Osten, Digitale Verarbeitung und Auswertung von Interferenzbildern (Akademie Verlag, Berlin, 1991).

79. H. O. Peitgen and D. Saupe, The Science of Fractal Images (Springer-Verlag, New York, 1988).

80. A. P. Pentland, Fractal-based description of natural scenes, IEEE Trans. Pattern Anal. Mach. Intell. 6 (1984) 661-674.

81. H. Qin and D. Terzopoulos, D-NURBS: A physics-based framework for geometric design, IEEE Trans. Visual. Comput. Graph. 2(1) (1996) 85-96.

82. D. Reisfeld, Constrained phase congruency: Simultaneous detection of interest points and of their scales, Computer Vision and Pattern Recognition, Proceedings CVPR '96 (IEEE Computer Society, 1996).

83. G. Rilling, P. Flandrin and P. Gonçalvès, On empirical mode decomposition and its algorithms, in Proc. IEEE EURASIP Workshop Nonlinear Signal Image Processing, Grado, Italy (2003).

84. http://www.ks.informatik.uni-kiel.de/visatec 
85. D. Shepard, A two dimensional interpolation function for irregularly spaced data, in Proc. ACM 23rd National Conference (1968), pp. 517-524.

86. G. Sommer, T. Bülow and D. Pallek, Riesz transforms for the isotropic estimation of the local phase of moire interferograms, in Symposium für Mustererkennung, DAGM 2000, eds. Ch. Perwass, G. Sommer and N. Krüger, Vol. 22 (Springer-Verlag, Kiel, 2000), pp. 333-340.

87. H. Stark, An extension of the Hilbert transform product theorem, Proc. IEEE 59 (1971) 1359-1360.

88. E. Stein and G. Weiss, Introduction to Fourier Analysis on Euclidian Spaces (Princeton University Press, New Jersey, 1971).

89. C. W. Stewart et al., Fractional Brownian motion models for synthetic aparture radar imagery scene segmentation, Proc. IEEE 81 (1993) 1511-1522.

90. M. S. Taqqu, V. Teverovsky and W. Willinger, Estimators for long range dependence: An empirical study, Fractales 3 (1995) 785-788.

91. M. Unser, Splines: A perfect fit for signal and image processing, IEEE Signal Process. Magazine 11 (1999) 22-38.

92. M. Unser, A. Aldroubi and M. Eden, B-spline signal processing: Part I-theory, IEEE Trans. Signal Process. 41(2) (1993) 821-833.

93. M. Unser, A. Aldroubi and M. Eden, B-spline signal processing: Part II-efficient design and applications, IEEE Trans. Signal Process. 41(2) (1993) 821-833.

94. L. Vincent, Morphological grayscale reconstruction in image analysis: Applications and efficient algorithms, Technical report 91-16, Harvard Robotics Laboratory, November 1991. IEEE Trans. Image. Process. 2(2) (1993) 176-201.

95. G. W. Wornell, Wavelet-based representation for the $1 / \mathrm{f}$ family of fractal process, Proc. IEEE 81(10) (1993) 1428-1450.

96. G. W. Wornell and A. V. Oppenheim, Estimation of fractal signals from noisy measurements using wavelets, IEEE Trans. Signal Process. 40 (1992) 611-623.

97. Z. Yang, D. Qi and L. Yang, Signal period analysis based on Hilbert-Huang transform and its application to texture analysis, (Preprint), http://www.ims.nus.edu.sg/ preprints/2004-38.pdf

98. N. Yokoya, K. Yamamoto and N. Funakubo, Fractal-based analysis and interpolation of 3D natural surface shapes and their application to terrain modeling, Comput. Vis. Graph. Image Process. 46 (1989) 284-302.

99. Z. Wu and N. E. Huang, A study of the characteristics of white noise using the empirical mode decomposition method, Proceedings: Math. Phys. Eng. Sci. 460(2046) (2004) 1597-1611.

100. J. Zhou, N. M. Patrikalakis, S. T. Tuohy and X. Ye, Scattered data fitting with simplex splines in two and three dimensional spaces, The Visual Computer 13(7) (1997) 295-315. 